\title{
Appearance of deterministic mixing behavior from ensembles of fluctuating hydrodynamics simulations of the Richtmyer-Meshkov instability
}

\author{
Kiran Narayanan* and Ravi Samtaney ${ }^{\dagger}$ \\ Mechanical Engineering Program, King Abdullah University of Science and Technology, Thuwal 23955 6900, Saudi Arabia
}

(Received 1 December 2017; published 19 April 2018)

\begin{abstract}
We obtain numerical solutions of the two-fluid fluctuating compressible Navier-Stokes (FCNS) equations, which consistently account for thermal fluctuations from meso- to macroscales, in order to study the effect of such fluctuations on the mixing behavior in the Richtmyer-Meshkov instability (RMI). The numerical method used was successfully verified in two stages: for the deterministic fluxes by comparison against air-SF 6 RMI experiment, and for the stochastic terms by comparison against the direct simulation Monte Carlo results for He-Ar RMI. We present results from fluctuating hydrodynamic RMI simulations for three He-Ar systems having length scales with decreasing order of magnitude that span from macroscopic to mesoscopic, with different levels of thermal fluctuations characterized by a nondimensional Boltzmann number (Bo). For a multidimensional FCNS system on a regular Cartesian grid, when using a discretization of a space-time stochastic flux $Z(x, t)$ of the form $Z(x, t) \rightarrow$ $1 / \sqrt{h \triangle t} N(i h, n \Delta t)$ for spatial interval $h$, time interval $\Delta t, h$, and Gaussian noise $N$ should be greater than $h_{0}$, with $h_{0}$ corresponding to a cell volume that contains a sufficient number of molecules of the fluid such that the fluctuations are physically meaningful and produce the right equilibrium spectrum. For the mesoscale RMI systems simulated, it was desirable to use a cell size smaller than this limit in order to resolve the viscous shock. This was achieved by using a modified regularization of the noise term via $Z(x, t) \rightarrow 1 / \sqrt{\Delta t \max \left(h^{3}, h_{0}^{3}\right)} N(i h, n \Delta t)$, with $h_{0}=\xi h \forall h<h_{0}, \xi \in \mathbb{Z}^{+}$. Our simulations show that for systems with Bo $\ll 1$ deterministic mixing behavior emerges as the ensemble-averaged behavior of several fluctuating instances, whereas when $\mathrm{Bo} \approx 1$, a deviation from deterministic behavior is observed. For all cases, the FCNS solution provides bounds on the growth rate of the amplitude of the mixing layer.
\end{abstract}

DOI: 10.1103/PhysRevE.97.043111

\section{INTRODUCTION}

Thermal fluctuations are the manifestation of jiggling of the microscopic constituents of a fluid; traditional deterministic governing equations of fluid mechanics such as the compressible Navier-Stokes (CNS) equations describe macroscopic hydrodynamic systems where the probabilistic effects of such spontaneous, random fluctuations are negligible. However, there are systems in which thermal fluctuations can drive the macroscopic physical phenomenon, thereby necessitating that one account for them in the equations governing fluid flow. Examples include instabilities at fluid-fluid interfaces where they affect the flow both quantitatively and qualitatively [1], systems with small length scales such as flow in nanochannels [2], rarified gas flows where the mean free path of the gas is comparable to the characteristic length of the flow [3], microgravity flows where they can lead to development of giant fluctuations [4], and multiscale simulation methods for fluids that employ algorithmic refinement by coupling a particle description to a continuum description of the system by including fluctuations in the continuum model at the handshake region [5-7]. Particle methods such as molecular dynamics (MD) capture naturally the thermal fluctuations albeit at a high computational cost, which makes them unfeasible as a

\footnotetext{
*Kiran.Narayanan@kaust.edu.sa

${ }^{\dagger}$ Ravi.Samtaney@kaust.edu.sa
}

computational tool for modeling large systems. In contrast, fluctuating hydrodynamics relies on governing equations that incorporate the effect of thermal fluctuations via the addition of the divergence of a stochastic flux to the otherwise deterministic fluid equations. The resulting system of stochastic partial differential equations is a coarse-grained representation of microscopic dynamics [8], constitutes a mesolevel model of the system, and provides a compromise between computational cost and the ability to capture thermal fluctuations. The stochastic flux that was originally postulated by Landau and Lifshitz $[9,10]$ was derived for equilibrium systems by several approaches [11-13] and was also extended to mixtures [14-16]. Their validity for nonequilibrium systems was derived by Español [8] and verified by particle simulations [17-19].

Coarse graining is an averaging process that reduces the number degrees of freedom (DOF) (also called relevant variables) in the evolution equation for the system so as to resolve only the physics of interest and keep the numerical cost associated with the solution of the governing equations low. See the notes in [20] for details of the coarse graining of the dynamics of a fluid based on separation of time scales. In short, there are different levels of coarse graining, with decreasing DOF, and although a given level can resolve the physics accurately for all time scales above its minimum limit, the use of a high-DOF model to represent physics that only involves much larger time scales is computationally expensive. The fluctuating compressible Navier-Stokes (FCNS) system, in particular, is a coarse-grained model of the Hamiltonian equations of motion 
that describe the evolution of the microscopic constituents of the fluid, can successfully resolve temporal scales greater than the acoustic time for sound wave propagation, and can be used to consistently include thermal fluctuations up to the macroscopic scales of continuum thermodynamics [21].

Here, we include thermal fluctuations consistently up to the macroscales by considering the two-fluid FCNS equations, so as to use numerical solutions of FCNS equations to study the qualitative and quantitative effect of thermal fluctuations on instability growth for the Richtmyer-Meshkov instability (RMI). RMI occurs when a fluid-fluid interface is impulsively accelerated by a shock. The Rayleigh-Taylor instability (RTI) is another example of an instability that occurs at the fluidfluid interface in the presence of gravity. As reviewed in the literature [22-24], in both situations experimental, theoretical, and simulation-based results have tried to explain the complex mechanisms of fluid mixing that occur. The growth of the mixing layer is of central importance and it is understood that for RMI there are two stages of mixing. The first stage is characterized by a linear phase with corresponding linear growth of perturbations corresponding to the most unstable wavelength $\lambda_{m}$. The second stage is that of nonlinear growth followed by turbulent mixing at the interface.

Our motivation, approach, and objectives for the work presented here are derived from the role of thermal fluctuations in RTI-induced mixing and by extension in RMI-induced mixing, which is considered to be significant in three ways. First, it is believed that they trigger the onset of the first stage, i.e., they govern the mechanisms that initiate the instability leading to the linear growth of the interface. MD simulations of RTI have demonstrated that, at least for equally sized fluid particles, a long startup is observed wherein there are spontaneous fluctuation-driven sites of mixing [25]. Continuum simulation approaches that lack thermal fluctuations induce the instability by perturbing the interface with a spectrum, whereas MD simulations start with a flat interface after which atomistic motion triggers the instability. The former generally yields growth rate values that are about half those of observed experiments when only short-wavelength modes are used [26], whereas the latter has shown to produce better results owing to the capture of perturbations at all wave numbers [1], thus emphasizing the need to include thermal fluctuations. Second, fluctuations may play a key role during the final stage of mixing by manifesting nucleation sites for the breakup of large flow features such as spikes that lead to smaller features [24], which have been observed experimentally [27] and predicted successfully by the direct simulation Monte Carlo (DSMC) method that includes thermal fluctuations [1], in contrast to CNS-based macroscopic models that have not. Third, it is has been shown, at least for RTI using MD simulations, that the deterministic predictions are ensemble-averaged outcomes of underlying microscopic processes that occur across multiple scales, even for system lengths as small as $1 \mu \mathrm{m}$ [28]. Such an emergence of macroscopic behavior from microscopic dynamics is of importance in the field of nanotechnology.

Various numerical methods for fluctuating hydrodynamics have been developed for the fully compressible formulation and simplifications thereof. Studies in one dimension include [29-31]. Balboa et al. [32] presented several numerical schemes for the isothermal compressible and incompressible equations and used it to study large concentration fluctuations in microgravity. Bell et al. [33] used a finite volume method for the FCNS system for a binary mixture of ideal gases and applied it to Rayleigh-Bénard convection and RTI. The FCNS system we consider in our work is fully compressible; the energy equation with varying temperature is included in the system. Balakrishnan et al. [34] developed a finite volume method for an identical system of equations for multiple species, which they used to study diffusion-driven RTI. The extension of their work to reactive mixtures by Bhattacharjee et al. [35] was motivated by application to turbulent flame fronts and instabilities therein. Our simulation-based study is motivated by applications to flow instabilities in the context of RMI. We found in the literature only particle-based studies of RMI that naturally accounted for thermal fluctuations, which includes the DSMC study in [36] and molecular dynamics studies of RMI in liquids such as the one presented in [37]. We performed fluctuating hydrodynamic RMI simulations for three $\mathrm{He}-\mathrm{Ar}$ systems of length scales that differed by an order of magnitude as determined by the domain length: a system identical to the one in [36] of length scale $O\left(10^{-5}\right) \mathrm{m}$, and two other systems of length scale $O\left(10^{-6}\right) \mathrm{m}$ and $O\left(10^{-7}\right) \mathrm{m}$, respectively. Decreasing the system size by an order of magnitude each time enabled us to investigate a range of length scales that spanned between that which can be considered macroscopic and that which can be considered mesoscopic with at least theoretically increasing significance of thermal fluctuations.

The outline of the paper is as follows. Section II lists the governing equations for a two-fluid mixture of ideal gases. Section III describes the regularization of noise as used in the numerical method, while Appendix A presents all other numerical details. The problem setup for application of the numerical method to RMI and diagnostics used to quantify perturbation growth are described in Secs. IV A and IV B, respectively. We verify the deterministic portion of our numerical solver for the CNS equations against results from theory and the air- $\mathrm{SF}_{6}$ RMI experiment in [38] (this is summarized in Sec. IVD). Next, we verify FCNS-based results against DSMC results in [36] for a system that can be considered macroscopic. Here, we picked a DSMC solution as a benchmark because the method naturally captures stochasticity arising due to thermal fluctuations. Results of this stochastic verification are presented in Sec. IVE. Results of FCNS equations applied to RMI for the two systems of length scale leaning towards the mesoscale regime of $O\left(10^{-6}\right) \mathrm{m}$ and $O\left(10^{-7}\right)$ are presented in Secs. IV F and IV G, respectively. Section V presents a discussion of our results in terms of nondimensional parameters. Finally, Sec. VI presents the conclusion. Appendix B presents the verification of equilibrium static structure factors for the choice of $h_{0}$ for the He-Ar binary gas mixture at rest.

\section{GOVERNING EQUATIONS}

We consider a binary mixture of two perfectly miscible ideal gases denoted as species 1 and species 2 of molecular mass $m_{1}$ and $m_{2}$, respectively. We denote the total number of species in the mixture as $N_{s}=2$ and the set of species indices as $S=[1,2]$. The mass density of the mixture, $\rho$, is the sum of individual species densities, i.e., $\rho=\sum_{k \in S} \rho_{k}$. The mass fraction of species $k$ is $Y_{k}$, which is defined through $\rho_{k}=Y_{k} \rho$, 
with $\sum_{k \in S} Y_{k}=1$. There are $N_{S}-1=1$ independent species mass densities and therefore, for the binary mixture, we only consider the evolution of species 1 .

The fluctuating compressible Navier-Stokes equations for the mixture may be written in conservative form as

$$
\mathcal{U}_{t}+\nabla \cdot \mathcal{F}_{H}=\nabla \cdot \mathcal{F}_{G}+\nabla \cdot \mathcal{F}_{\mathcal{Z}},
$$

where $\mathcal{U}=\mathcal{U}(\mathbf{x}, t, \mathbf{N})$ is the vector of conserved quantities, namely, mass, momentum total energy, and mass of species 1 , and is given by $\mathcal{U}=\left(\rho, \rho \mathbf{u}, E, \rho Y_{1}\right)^{T} ; \mathbf{u}$ is the velocity and $E$ is the total energy. The addition of a stochastic flux term makes the equations a stochastic partial differential equation (SPDE) system and therefore $\mathcal{U}$ is a multivariate process. Here $\mathbf{x}$ is the spatial coordinate, $t$ is the time, and $\mathbf{N} \equiv \mathbf{N}(\mathbf{x}, t)$ is a Gaussian noise field which is formally the derivative of a Wiener sheet. $\mathcal{F}_{H}, \mathcal{F}_{G}$, and $\mathcal{F}_{Z}$ are the hyperbolic or advective flux, diffusive or dissipative flux, and stochastic or fluctuating flux, respectively. At equilibrium, a fluctuationdissipation condition is satisfied when the system has reached a statistical steady state. It should be noted that the stochastic forcing in Eq. (1) is the divergence of white noise that models conservative, intrinsic, i.e., thermal, fluctuations. We have that

$$
\mathcal{F}_{H}=\left(\begin{array}{c}
\rho \mathbf{u} \\
\rho \mathbf{u u}+P \mathbf{I} \\
(E+P) \mathbf{u} \\
\rho \mathbf{u} Y_{1}
\end{array}\right), \quad \mathcal{F}_{G}=\left(\begin{array}{c}
0 \\
\boldsymbol{\tau} \\
\boldsymbol{\tau} \cdot \mathbf{u}+\mathbf{q}+G \mathbf{f}_{1} \\
\mathbf{f}_{1}
\end{array}\right),
$$

and the stochastic flux is given by

$$
\mathcal{F}_{Z}=\left(\begin{array}{c}
0 \\
\tilde{\boldsymbol{\tau}} \\
\mathbf{u} \cdot \tilde{\boldsymbol{\tau}}+\underset{\mathbf{q}}{\tilde{\mathbf{f}}_{1}}+G \tilde{\mathbf{f}}_{1}
\end{array}\right)
$$

Here, $\boldsymbol{\tau}$ is the shear stress, $\mathbf{q}$ is the heat flux vector, $\mathbf{f}_{1}$ is the barycentric diffusion flux with $\mathbf{f}_{1}+\mathbf{f}_{2}=\mathbf{0} ; \tilde{\boldsymbol{\tau}}$ and $\tilde{\mathbf{q}}$ are the stochastic stress tensor and heat flux, respectively; $P$ is the thermodynamic pressure; and $\tilde{\mathbf{f}}_{1}$ is the stochastic diffusion flux of species 1 with $\tilde{\mathbf{f}}_{1}+\tilde{\mathbf{f}}_{2}=0$. Also, for a binary mixture of ideal gases we have that $[33,39]$

$$
G=S_{T} \frac{k_{B} T}{\left(m_{1} Y_{2}+m_{2} Y_{1}\right)}+\left(C_{p 1}-C_{p 2}\right) T .
$$

\section{A. Thermodynamics}

The pressure $P$ is given by

$$
P=\rho R_{u} T \sum_{k=1}^{N_{s}} \frac{Y_{k}}{w_{k}},
$$

where $R_{u}=k_{B} N_{A}$ is the universal gas constant, where $k_{B}$ is Boltzmann's constant and $N_{A}$ is Avogadro's number; $w_{k}=$ $m_{k} N_{A}$ is the molecular weight of species $k$, where $m_{k}$ is the molecular mass of that species. The total energy is

$$
E=\rho e\left(T, Y_{k}\right)+\frac{1}{2}|\mathbf{u}|^{2},
$$

where the internal energy per unit mass of the mixture is $e\left(T, Y_{k}\right)=\sum_{k=1}^{N_{s}} Y_{k} e_{k}(T)$, where $e_{k}=C_{v, k} T, C_{v, k}$ being the specific heat for constant volume of species $k$. The enthalpy per unit mass of the mixture is given by $h\left(T, Y_{k}\right)=\sum_{k=1}^{N_{s}} Y_{k} h_{k}(T)$, where $h_{k}=e_{k}+R_{u} T / w_{k}$. The sound speed of the mixture is $c=\sqrt{\gamma P / \rho}$.

\section{B. Deterministic diffusion fluxes}

It can be shown that for the binary mixture of ideal gases that

$$
\mathbf{f}_{1}=\rho D\left(\nabla Y_{1}+S_{T} Y_{1} Y_{2} \frac{\nabla T}{T}+\left(X_{1}-Y_{1}\right) \frac{\nabla P}{P}\right),
$$

where the Soret coefficient $S_{T}$ is usually assumed to be independent of concentration [33,39] and $D$ is the diffusion coefficient. The heat flux vector $\mathbf{q}$ is given by $\mathbf{q}=\kappa \nabla T$. The shear stress tensor $\tau$ is given by

$$
\boldsymbol{\tau}=\eta\left(\nabla \mathbf{u}+\nabla \mathbf{u}^{T}\right)+\left(\zeta-\frac{2}{3} \eta\right)(\nabla \cdot \mathbf{u}) \mathbf{I},
$$

where $\eta$ is the dynamic shear viscosity coefficient and $\zeta$ is the bulk viscosity coefficient. All transport coefficients are evaluated using the mass fraction and temperature-dependent relationships for a binary gas mixture given in [39] that are also summarized in the Appendix of [33].

\section{Stochastic fluxes}

$\tilde{\boldsymbol{\tau}}, \tilde{\mathbf{q}}$, and $\tilde{\mathbf{f}}_{k}$ are Gaussian random fields with zero mean, i.e., $\langle\tilde{\boldsymbol{\tau}}\rangle=0,\langle\tilde{\mathbf{q}}\rangle=0$, and $\left\langle\tilde{\mathbf{r}}_{k}\right\rangle=0$ where $\langle\cdot\rangle$ denotes a suitable defined ensemble average. The cross correlations are zero; i.e., $\langle\tilde{\boldsymbol{\tau}}, \tilde{\mathbf{q}}\rangle=\left\langle\tilde{\boldsymbol{\tau}}, \tilde{\mathbf{f}}_{k}\right\rangle=\left\langle\tilde{\mathbf{q}}, \tilde{\mathbf{f}}_{k}\right\rangle=0$. The stochastic stress tensor can be written as

$$
\tilde{\boldsymbol{\tau}}(\mathbf{x}, t)=\sqrt{2 k_{B} \eta T} \tilde{\mathbf{N}}^{s}+\left(\sqrt{\frac{k_{B} \zeta T}{3}}-\sqrt{\frac{2 k_{B} \eta T}{3}}\right) \operatorname{Tr}\left(\tilde{\mathbf{N}}^{s}\right) \mathbf{I},
$$

where $k_{B}$ is Boltzmann's constant, and $\tilde{\mathbf{N}}^{s}(\mathbf{x}, t)=$ $\left(\mathbf{N}^{s}(\mathbf{x}, t)+\left(\mathbf{N}^{s}(\mathbf{x}, t)\right)^{T}\right) / \sqrt{2}$ is a symmetric Gaussian random tensor field; the $\sqrt{2}$ in the denominator accounts for the reduction in variance due to averaging. The covariance of $\mathbf{N}^{s}(\mathbf{x}, t)$ is given by $\left\langle N_{i j}^{s}(\mathbf{x}, t) N_{k l}^{s}\left(\mathbf{x}^{\prime}, t^{\prime}\right)\right\rangle=$ $\delta_{i j}^{K} \delta_{k l}^{K} \delta\left(\mathbf{x}-\mathbf{x}^{\prime}\right) \delta\left(t-t^{\prime}\right)$, i.e., it has zero mean and variance equal to 1 , where $\delta$ is the Dirac delta and $\delta^{K}$ is the Kronecker delta. The stochastic diffusion flux of the $k$ th species is given by

$$
\tilde{\mathbf{f}}_{1}=\sqrt{2 D \rho\left[Y_{1} Y_{2}\left(m_{1} Y_{2}+m_{2} Y_{1}\right)\right]} \mathbf{N}^{Y_{1}},
$$

where $\mathbf{N}^{Y_{1}}(\mathbf{x}, t)$ is such that $\left\langle N_{i}^{Y_{1}}(\mathbf{x}, t) N_{j}^{Y_{1}}\left(\mathbf{x}^{\prime}, t^{\prime}\right)\right\rangle=$ $\delta_{i j}^{K} \delta\left(\mathbf{x}-\mathbf{x}^{\prime}\right) \delta\left(t-t^{\prime}\right)$

The stochastic heat flux vector is a can be written as

$$
\tilde{\mathbf{q}}(\mathbf{x}, t)=T \sqrt{2 k_{B} \kappa} \mathbf{N}^{q},
$$

where $\mathbf{N}^{q}(\mathbf{x}, t)$ is a white-noise random Gaussian vector field, whose covariance is given by $\left\langle N_{i}^{q}(\mathbf{x}, t) N_{j}^{q}\left(\mathbf{x}^{\prime}, t^{\prime}\right)\right\rangle=$ $\delta_{i j}^{K} \delta\left(\mathbf{x}-\mathbf{x}^{\prime}\right) \delta\left(t-t^{\prime}\right)$.

\section{REGULARIZATION OF NOISE}

We discretize the solution to Eq. (A2) in time and space, approximating $\mathcal{U}$ by the values at cell centers of the Cartesian grid, i.e., $\mathcal{U}=\mathbf{U}_{\mathbf{i}}^{n} \approx \mathrm{U}\left(\mathbf{i} h, n \triangle t, \mathbf{N}_{\mathbf{i}}^{n}\right)$, where $\mathbf{i} \in \mathrm{Z}^{\mathrm{D}}$ denotes cell centers, $h$ is the mesh spacing, $D=2$ is the dimensionality 
of space, $n$ is the time step index, $\Delta t$ is the time step, and $\mathbf{N}_{\mathbf{i}}^{n}$ is the spatiotemporal discretization of the noise $\mathbf{N} \equiv \mathbf{N}(\mathbf{x}, t)$. The details of the numerical method used are presented in Appendix A. In brief, we use discretizations that are deterministically second order in space, and the Euler-Maruyama scheme for time integration. Here, we focus on the discretization of the noise terms and their regularization thereof. The finite volume method naturally deals with the delta-correlated fluctuating quantities in Sec. II C. As derived by Chandrasekhar [40], integration schemes for stochastic partial differential equations commonly regularize the Dirac delta's appearing in the expression for the stochastic terms. For time, we have

$$
\delta\left(t_{1}-t_{2}\right) \rightarrow \frac{\delta_{t_{1} t_{2}}}{\Delta t},
$$

where $t_{p}=t^{n+(p-1)}, p=(1,2), t^{n}=n \Delta t$. For space, the analogous regularization is

$$
\delta\left(\mathbf{x}_{\mathbf{i}}-\mathbf{x}_{\mathbf{j}}\right) \rightarrow \delta_{\mathbf{i j}} / h^{3},
$$

instead of which we propose a modified regularization; the rationale for such a regularization and its description are as follows. It is important to keep in mind that the governing equations presented in Sec. II are to be interpreted as a coarse-grained representation of microscopic dynamics [8]. The temporal and spatial coarse-graining scales do not appear explicitly in the governing equations as they are written in Sec. II; however, this can be done if desired (see [41]). Where they do appear explicitly is in the temporal and spatial discretization of the governing equations through the time step size $\Delta t$ and the grid spacing $h$, i.e., the cell volume $h^{3}$, or more precisely the number of fluid molecules per cell. For ease of exposition, let us consider the single-species case and thereby ignore the fourth conservation law for mass flux of species 1 . Let us also, for now, only consider the spatial coarse graining. The discrete conserved field $\mathbf{U}_{\mathbf{i}}$ which is a stochastic variable approximates the behavior of the true atomistic configuration over long length scales $h$ and time scales $\Delta t$ compared to atomistic scales, in the sense of certain integral averages. For sufficiently large finite volume cells, the integral of $\mathbf{U}_{\mathbf{i}}$ over the cell corresponds to the total particle mass, momentum, and sum of particle potential and kinetic energies of the particles contained inside the cell. This can be expressed mathematically as

$$
\begin{aligned}
\int_{V_{\mathbf{i}}} \mathbf{U} d V & =V_{\mathbf{i}}\langle\mathbf{U}\rangle_{\mathbf{i}}=V_{\mathbf{i}}\left[\begin{array}{c}
\rho \\
\rho \mathbf{u} \\
E
\end{array}\right]_{\mathbf{i}} \\
& \cong \sum_{\mathbf{i}}\left[\begin{array}{c}
m_{\mathbf{i}} \\
\mathbf{p}_{\mathbf{i}} \\
e_{\mathbf{i}}^{\text {tot }}
\end{array}\right] \chi_{\mathbf{i}}\left(\mathbf{x}-\mathbf{x}_{i}(t)\right),
\end{aligned}
$$

where $m_{\mathbf{i}}, \mathbf{p}_{\mathbf{i}}$, and $e_{\mathbf{i}}^{\text {tot }}$ are the mass, momentum, and energy of the particles (for a simple fluid, atoms), respectively; $\chi_{\mathbf{i}}$ is the characteristic function of cell $\mathbf{i}$. Dividing through by $V_{\mathbf{i}}$, we get that

$$
\langle\mathbf{U}\rangle_{\mathbf{i}} \cong \sum_{\mathbf{i}}\left[\begin{array}{c}
m_{\mathbf{i}} \\
\mathbf{p}_{\mathbf{i}} \\
e_{\mathbf{i}}
\end{array}\right] \bar{\delta}\left(\mathbf{x}-\mathbf{x}_{i}(t)\right),
$$

where $\bar{\delta}\left(\mathbf{x}-\mathbf{x}_{i}(t)\right)=\chi_{\mathbf{i}}\left(\mathbf{x}-\mathbf{x}_{i}(t)\right) / V_{\mathbf{i}}$ is the coarse-graining operator. The coarse graining is therefore valid as mentioned earlier, for sufficiently large volumes; i.e., it should contain a sufficiently large number of particles. When using the regularization in Eq. (13), if the discretization is such that the cell volume $h^{3}$ is not sufficiently large, the fluctuations become very large, leading to unphysical numerical artifacts. The choice of the correct grid size $h$ to be used in fluctuating hydrodynamics simulations has been in the past based on two approaches. For systems with gases operating near the rarefied region, a cell volume $h^{3}$ that contains at least an order of a few hundred particles has been suggested [29]. A more systematic approach is to choose $h$ such that the equilibrium static structure factors match theoretical values [31]. This latter approach is used by most authors presently. Let us denote this value of $h$ (the one that produces correct static structure factors) as $h_{0}$. The regularization in Eq. (13) prohibits the use of a grid spacing finer, i.e., lesser than $h_{0}$, which, though undesirable for the stochastics, may be desired to capture some essential physics of the problem being studied. We encountered such a situation in our study of the RMI problem for the domain sizes of systems considered in Secs. IVF and IV G, for which resolving the viscous shock meant using $h<h_{0}$. This motivated us to propose a regularization of the form

$$
\delta\left(\mathbf{x}_{\mathbf{i}}-\mathbf{x}_{\mathbf{j}}\right) \rightarrow \frac{\delta_{\mathbf{i j}}}{\max \left(h^{3}, h_{0}^{3}\right)},
$$

with $h_{0}$ being an integer multiple of $h$ whenever $h<h_{0}$, which we use in the present work. The determination of $h_{0}$ for the binary gas mixture of helium and argon via verification of equilibrium static structure factors is presented in detail in Appendix B.

\section{APPLICATION TO RICHTMYER-MESHKOV INSTABILITY}

RMI occurs when the interface between two fluids with different densities is accelerated impulsively, usually by a moving shock wave passing through the interface. The driving mechanism for the instability is the baroclinic vorticity deposition at the interface because of a misalignment of the gradients of pressure and density. The direction of the deposited vorticity dictates the acceleration of the interface [42]. The perturbed interface is considered as a combination of bubbles and spikes; the light fluid penetrating into the heavier fluid is a bubble and heavier fluid penetrating into the light fluid constitutes a spike. The occurrence of RMI was first theoretically established using linear stability analysis by Richtmyer [43] and later experimentally verified by Meshkov [44]. RMI is of importance in technological applications such as scramjet engines and inertial confinement fusion, and in astrophysical phenomena such as supernova blast waves interacting with surrounding matter. Richtmyer's analysis dealt with the interaction of a shock wave with a perturbed contact discontinuity that separated gases of different densities and concluded that the perturbations grew linearly in time. Nonlinear analysis has revealed that the perturbations grow as a power law in time [22]. 


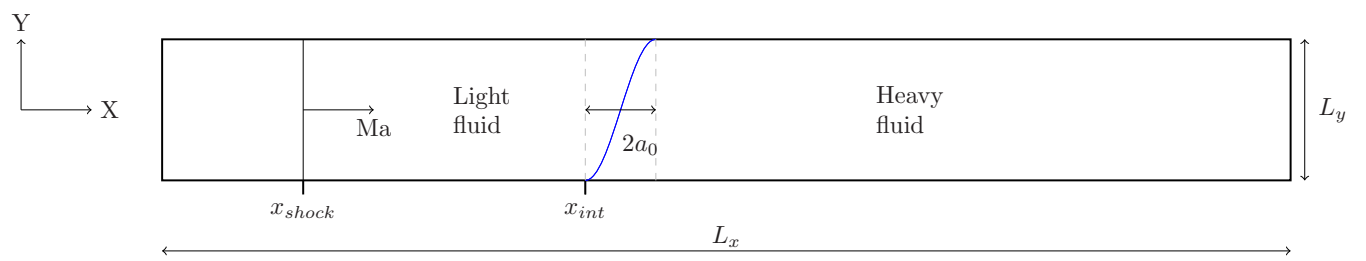

FIG. 1. RMI simulation setup.

\section{A. Simulation setup}

For all the RMI simulations presented here, we consider a rectangular computational domain $\left[0, L_{x}\right] \times\left[-L_{y} / 2, L_{y} / 2\right]$ with X-direction length $L_{x}$ and Y-direction length $L_{y}$. The mesh spacing in both the $\mathrm{X}$ and $\mathrm{Y}$ directions is $h$ and the number of cells in the $\mathrm{X}$ and $\mathrm{Y}$ directions are $n_{x}$ and $n_{y}$, respectively. A schematic of the simulation setup is shown in Fig. 1. Species 1 is the light fluid and species 2 is the heavy fluid; the light fluid is on the left side of the interface, with the $\mathrm{X}$ location of the interface denoted by $x_{\text {int }}$. A viscous shock is located in the light fluid region at the $\mathrm{X}$ location denoted by $x_{\text {shock }}$. It was ensured that the viscous shock profile was fully resolved. For the deterministic runs the fluid-fluid interface was perturbed with initial perturbation amplitude $a_{0}$ and wave number $k=2 \pi / \lambda_{p}$ with wavelength $\lambda_{p}$. The pre- and post-shock Atwood number are denoted $A_{t}^{-}$and $A_{t}^{+}$, respectively. They are defined as $A_{t}^{-}=$ $\left(\rho_{2}^{-}-\rho_{1}^{-}\right) /\left(\rho_{2}^{-}+\rho_{+}^{-}\right)$and $A_{t}^{+}=\left(\rho_{2}^{+}-\rho_{1}^{+}\right) /\left(\rho_{2}^{+}+\rho_{1}^{+}\right)$. SI units are used throughout. The parameters during the initial setup for the different RMI systems simulated in this study are listed in Table I.

\section{B. Diagnostics to quantify RMI evolution}

The parameters we use to quantify RMI evolution are described here. The mixing length $l_{m}$ is defined as the distance between spike and bubble tip locations, which are $l_{b}(t)$ and $l_{s}(t)$, respectively; i.e., $l_{m}(t)=l_{b}(t)-l_{s}(t)$. The amplitudes of spikes and bubbles are denoted $a_{s}$ and $a_{b}$, respectively, which are the distance from the unperturbed interface location $l_{\text {int }}$ to spike or bubble tip, i.e., $a_{b}(t)=l_{b}(t)-l_{\text {int }}(t)$ and $a_{s}(t)=$ $l_{s}(t)-l_{\text {int }}(t)$. The mean amplitude of the mixing layer is $a_{m}=\left(a_{b}+a_{s}\right) / 2=l_{m} / 2$. The calculation of $l_{b}(t)$ and $l_{s}(t)$ during each time step of the simulation uses thresholds on the spanwise spatial average of the mass fraction of species $1, Y_{1}$, which is defined as

$$
\bar{Y}_{1}(\mathbf{x}, t)=\frac{1}{L_{y}} \int_{0}^{L_{y}} Y_{1}(\mathbf{x}, t) d y .
$$

Positions $l_{b}(t)$ and $l_{s}(t)$ are identified as the $\mathrm{X}$ position where $\bar{Y}_{1} \leqslant 1-\epsilon$ and $\bar{Y}_{1} \geqslant \epsilon$, respectively, with $\epsilon=0.01$. The corresponding $X$ velocities are identified as bubble and spike velocities $v_{b}(t)$ and $v_{s}(t)$, respectively. Then, $l_{m}(t)$ and $a_{m}(t)$ are calculated using the relations given above. The growth rates of bubble and spike amplitudes are calculated as $d a_{b} / d t=v_{b}(t)$ and $d a_{s} / d t=v_{s}(t)$, and the growth rate of the interface amplitude as $d a_{m} / d t=\left(d a_{b} / d t+d a_{s} / d t\right) / 2$. The nondimensional time is defined as $\tau=k v_{0} t$, where $v_{0}=$ $k \Delta u A_{t}^{+} a_{0}^{+}$is the Richtmyer velocity where $\Delta u$ is the velocity jump across the interface, and the nondimensional amplitude is defined as $a_{m}^{*}=k\left(a_{m}-a_{0}^{+}\right)$, where $a_{0}^{+}$is the postshock amplitude. $\Delta u$ was calculated using standard equations for one-dimensional shock-wave propagation in a perfect gas [45].

For FCNS simulations, the ensemble-averaged growth rate denoted as $\left\langle d a_{m} / d t\right\rangle$ was obtained from the number of simulation instances, $n_{\text {samp }}$. The curves representing the bounds shown in Figs. 8, 12, and 15 represent the extrema at each time step from among all instances.

\section{Growth rate models}

Here, we provide the empirical expressions for the RMIinduced growth of a single-mode perturbation from some of the models in the literature that we compare in the forthcoming sections to our numerical results. A review of these models is

TABLE I. System parameters specified at the start of the simulation (all units in SI).

\begin{tabular}{|c|c|c|c|c|c|}
\hline & System 1 & System $2 \mathrm{a}$ & System $2 b$ & System 3 & System 4 \\
\hline Light fluid (species 1) & Air + Acetone & $\mathrm{He}$ & $\mathrm{He}$ & $\mathrm{He}$ & $\mathrm{He}$ \\
\hline Heavy fluid (species 2) & $\mathrm{SF}_{6}$ & $\mathrm{Ar}$ & $\mathrm{Ar}$ & $\mathrm{Ar}$ & $\mathrm{Ar}$ \\
\hline $\mathrm{Ma}$ & 1.21 & 1.5 & 1.5 & 2.0 & 2.0 \\
\hline$L_{x}$ & $78.022 \times 10^{-2}$ & $327.68 \times 10^{-6}$ & $350 \times 10^{-6}$ & $32.76 \times 10^{-6}$ & $16.38 \times 10^{-6}$ \\
\hline$L_{y}$ & $8.9 \times 10^{-2}$ & $24.9 \times 10^{-6}$ & $24.9 \times 10^{-6}$ & $4.09 \times 10^{-6}$ & $0.512 \times 10^{-6}$ \\
\hline$h$ & $0.0232 \times 10^{-2}$ & $1.6 \times 10^{-7}$ & $1.7 \times 10^{-7}$ & $1.6 \times 10^{-8}$ & $1.6 \times 10^{-8}$ \\
\hline$n_{x}$ & 3364 & 2048 & 2048 & 2048 & 1024 \\
\hline$n_{y}$ & 388 & 156 & 156 & 256 & 32 \\
\hline$x_{\text {int }}$ & $3.003 \times 10^{-2}$ & $2.457 \times 10^{-6}$ & $2.6 \times 10^{-6}$ & $0.3 L_{x}$ & $0.3 L_{x}$ \\
\hline$x_{\text {shock }}$ & $1.006 \times 10^{-2}$ & $6.144 \times 10^{-6}$ & $26.1 \times 10^{-6}$ & $0.25 L_{x}$ & $0.25 L_{x}$ \\
\hline$a_{0}$ & $0.2 \times 10^{-2}$ & $1.0 \times 10^{-6}$ & $1 \times 10^{-5}$ & $4.02 \times 10^{-7}$ & $5.12 \times 10^{-8}$ \\
\hline$\lambda_{p}$ & $5.933 \times 10^{-2}$ & $4.98 \times 10^{-5}$ & $4.98 \times 10^{-5}$ & $8.04 \times 10^{-6}$ & $1.024 \times 10^{-6}$ \\
\hline$k a_{0}$ & 0.21 & 0.126 & 1.26 & 0.31 & 0.31 \\
\hline$d t$ & $1.0 \times 10^{-7}$ & $4.5 \times 10^{-12}$ & $4.5 \times 10^{-12}$ & $1.2 \times 10^{-13}$ & $1.15 \times 10^{-13}$ \\
\hline
\end{tabular}


TABLE II. Coefficients for Sadot-type models of amplitude growth rate.

\begin{tabular}{lcc}
\hline \hline Model & $D$ & $E$ \\
\hline Sadot [46] & $1 \pm A_{t}^{+}$ & $3\left(1 \pm A_{t}^{+}\right) / 2\left(1 \pm A_{t}^{+}\right)$ \\
Niederhaus and Jacob [47] & $1 \pm A_{t}^{+}$ & $1 \pm A_{t}^{+}$ \\
Goncharov [48] & $1 \pm A_{t}^{+}$ & $3\left(1 \pm A_{t}^{+}\right) /\left(3+A_{t}^{+}\right)$ \\
Sohn [49] & $1 \pm A_{t}^{+}$ & $\left(2 \pm A_{t}^{+}\right) / 2$ \\
\hline \hline
\end{tabular}

provided in [22]. Richtmyer's [43] linear model for a shock in the light fluid moving into the interface with a heavy fluid, which is applicable when $k a_{0}<0.3$, is

$$
a_{m}(t)=a_{0}^{+}+v_{0} t .
$$

The family of two-dimensional nonlinear models based on the Sadot et al. [46] empirical model for the growth rate of the bubble and spike have the growth rate

$$
\frac{d a_{b / s}(t)}{d t}=v_{0} \frac{1+v_{0} k t}{1+D v_{0} k t+E v_{0}^{2} k^{2} t^{2}},
$$

where the coefficients $D$ and $E$ can be chosen according to different theories as summarized in Table II, where \pm denotes the value used for the bubble and spike, respectively.

\section{Verification of deterministic solver}

The numerical method and code for the deterministic solver (thermal fluctuations turned off) was first verified by direct comparison of results against the air- $\mathrm{SF}_{6} \mathrm{RMI}$ experiment in [38]. The air-acetone mixture was considered species 1 and $\mathrm{SF}_{6}$ was considered species 2 . The parameters of the boundary value problem were matched to the experiment and are given under System 1 in Table I. A $\gamma$ value of 1.271 was used for both the light and heavy fluids, the Atwood number $A_{t} \approx 0.6$, and the pre-shock and post-shock conditions are given under System 1 in Table III. Similar to the experiment in [38], the fluid-fluid interface perturbation was $\psi(y)=a_{0} \sin (k y)$, with initial perturbation amplitude $a_{0}=0.2 \mathrm{~cm}$, wave number $k=$ $2 \pi / \lambda$, and wavelength $\lambda_{p}=5.93333 \mathrm{~cm}$. For this simulation, the initial interface is a tanh profile of width $\delta \approx 15$ cells wide to avoid numerical artifacts. The left boundary had an inflow condition, the right wall was set to reflecting in the $\mathrm{X}$ direction, and periodic boundary conditions are imposed in the $Y$ direction.

Figures 2 and 3 show the comparison of experimentally observed and simulation-based interface location as a function

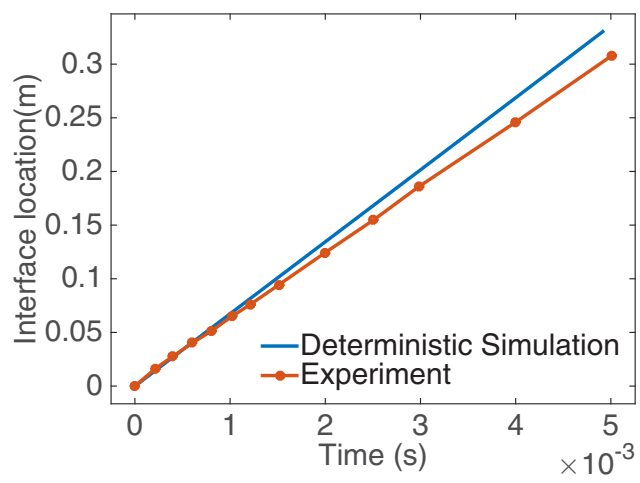

FIG. 2. Evolution of interface location for air-SF 6 RMI (System 1) compared to experimental result of Collins and Jacobs [38].

of time and nondimensional amplitude as a function of nondimensional time. We observe that our numerical method agrees well with the experiment even though our simulation is a viscous calculation. Viscous effects are negligible at early times for high Reynolds number flows at large scales because inertia plays the dominant role [50], and therefore agreement with experiment signifies the successful verification of our numerics. A qualitative comparison of the density plots obtained from the simulation and the density field from [38] is shown in Figs. 4 and 5. We find that the flow features pre- and post-shock are predicted well by the second order method for the advective and viscous terms developed in this work. Any late time differences are attributed to the fact that the actual experiment becomes three dimensional whereas the present simulations are two dimensional.

\section{E. Verification of fluctuating solver}

The numerical method for the two-fluid FCNS system was verified against the DSMC results in [36], where the authors consider several two-fluid systems and demonstrate that the DSMC system reproduces the universal behavior seen in two-fluid RMI, in that the DSMC-predicted metrics that have to do with the mixing zone, such as growth rate and amplitude, matched the results from Navier-Stokes-based simulations. They also simulated RMI in a domain size that was computationally feasible for a particle method such as DSMC. We pick their helium-argon Mach 1.5 case for our verification because of a twofold rationale. First, it enables one-on-one comparison with a numerical method that accounts for thermal fluctuations. Second, it enables the study of the emergence of

\begin{tabular}{|c|c|c|c|c|c|c|c|c|}
\hline & \multicolumn{2}{|c|}{ System 1} & \multicolumn{2}{|c|}{ System 2a } & \multicolumn{2}{|c|}{ System 3} & \multicolumn{2}{|c|}{ System 4} \\
\hline & Pre-shock & Post-shock & Pre-shock & Post-shock & Pre-shock & Post-shock & Pre-shock & Post-shock \\
\hline$a_{0}$ & $2.1 \times 10^{-3}$ & $2.0 \times 10^{-2}$ & $1.1 \times 10^{-6}$ & $2.766 \times 10^{-6}$ & $0.74 \times 10^{-6}$ & $1.46 \times 10^{-6}$ & $1.8 \times 10^{-7}$ & $2.5 \times 10^{-8}$ \\
\hline$\rho_{1}$ & 1.351 & 2.14 & 0.1785 & 0.368 & 0.1785 & 0.407 & 0.1785 & 0.404 \\
\hline$\rho_{2}$ & 6.804 & 10.7 & 1.7826 & 1.7826 & 1.7826 & 1.7826 & 1.7826 & 1.7826 \\
\hline$A_{t}$ & 0.6686 & 0.666 & 0.818 & 0.657 & 0.818 & 0.6 & 0.818 & 0.63 \\
\hline$P_{1}$ & $1.146 \times 10^{5}$ & $2.019 \times 10^{5}$ & $1.013 \times 10^{5}$ & $3.669 \times 10^{5}$ & $1.013 \times 10^{5}$ & $4.82 \times 10^{5}$ & $1.013 \times 10^{5}$ & $4.71 \times 10^{5}$ \\
\hline$P_{2}$ & $1.146 \times 10^{5}$ & $1.146 \times 10^{5}$ & $1.013 \times 10^{5}$ & $1.013 \times 10^{5}$ & $1.013 \times 10^{5}$ & $1.013 \times 10^{5}$ & $1.013 \times 10^{5}$ & $1.013 \times 10^{5}$ \\
\hline
\end{tabular}

TABLE III. Pre- and post-shock conditions for RMI simulations (All units in S.I.). The value of post-shock amplitude is the best-fit value. 


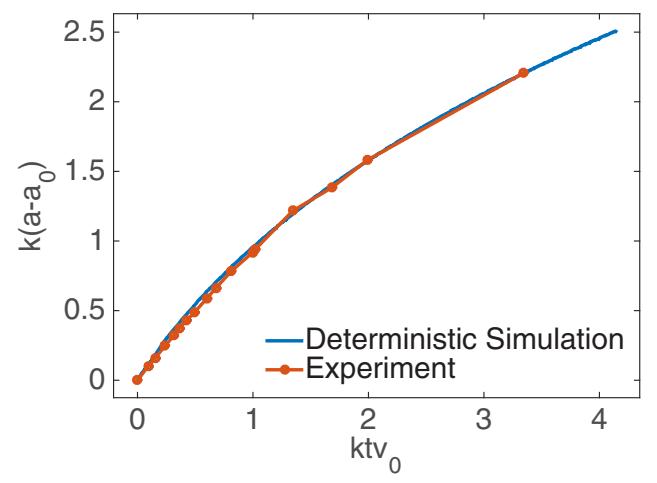

FIG. 3. Interface growth for air-SF $\mathrm{SF}_{6} \mathrm{RMI}$ simulation (System 1) compared to experimental result of Collins and Jacobs [38].

macroscopic behavior as the ensemble-averaged behavior of a system with microscopic fluctuations. This is because the physical length scale of the system as dictated by the domain size in the $\mathrm{Y}$ direction, which is half the wavelength of the perturbation $O\left(10^{-5}\right) \mathrm{m}$, and may be considered macroscopic. We match our simulation setup parameters to the experiment and consider helium as the light fluid (species 1) and argon as the heavier, species 2 . The system parameters are given under System 2a in Table I. The fluid-fluid interface perturbation was $\psi(y)=a_{0} \sin (k y)$, with initial perturbation amplitude $a_{0}=$ $1 \mu \mathrm{m}$, wave number $k=2 \pi / \lambda$, and wavelength $\lambda_{p}=50 \mu \mathrm{m}$. The left boundary had inflow condition, the right wall was set to reflecting in the $\mathrm{X}$ direction, and the lower and upper $\mathrm{Y}$ walls had reflecting condition in the $\mathrm{Y}$ direction because only half the sine wave was included in the perturbation. The results with fluctuations presented in this section are the averaged result from $N_{\text {samp }}=25$ runs, for each of which the maximum number of simulation time steps was limited to just after reshock owing to computational cost. This time step limit allowed us to investigate the linear part of the instability for the fluctuating case. The stand-alone deterministic simulation was run for longer times until and past reshock.

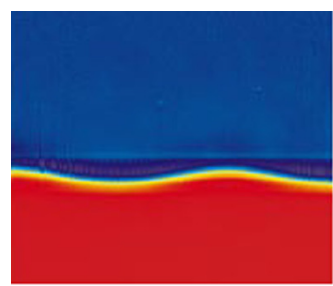

(a) $\mathrm{t}=0 \mathrm{~ms}$

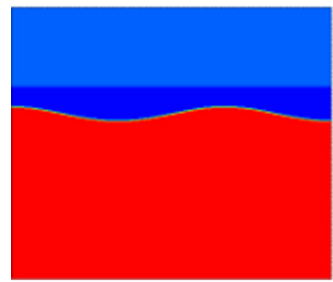

(e) $\mathrm{t}=0 \mathrm{~ms}$

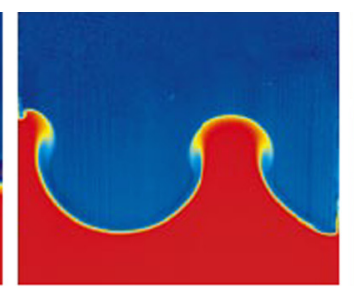

(b) $\mathrm{t}=2.5 \mathrm{~ms}$

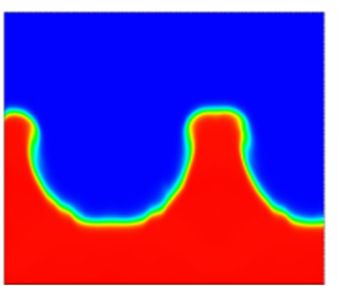

(f) $\mathrm{t}=2.5 \mathrm{~ms}$

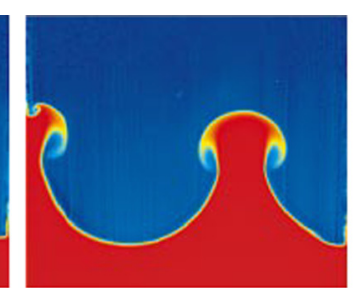

(c) $\mathrm{t}=3 \mathrm{~ms}$

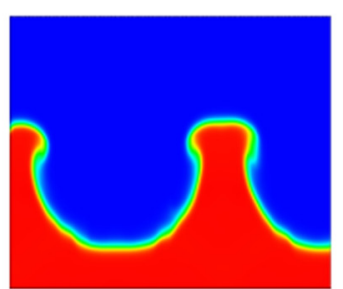

(g) $\mathrm{t}=3 \mathrm{~ms}$

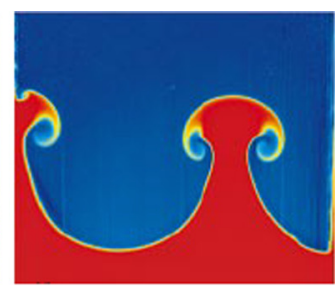

(d) $\mathrm{t}=4 \mathrm{~ms}$

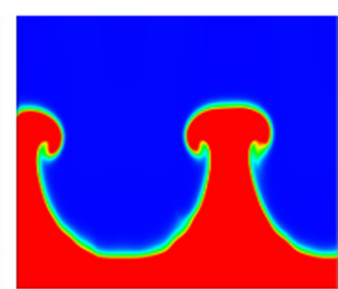

(h) $t=4 \mathrm{~ms}$

FIG. 4. Density plots for air-SF 6 RMI simulation (System 1) compared to experimental result of Collins and Jacobs [38]. 


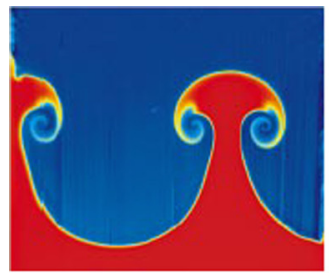

(a) $\mathrm{t}=5 \mathrm{~ms}$

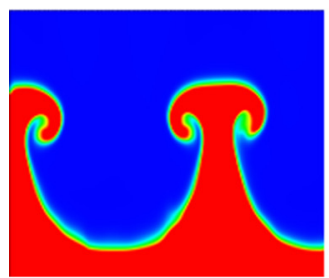

(e) $\mathrm{t}=5 \mathrm{~ms}$

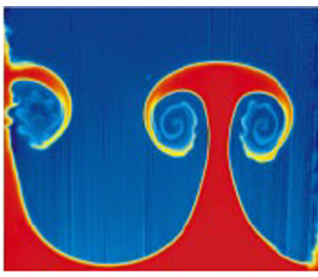

(b) $\mathrm{t}=6 \mathrm{~ms}$

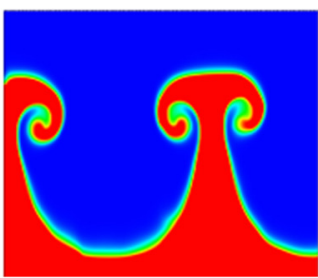

(f) $\mathrm{t}=6 \mathrm{~ms}$

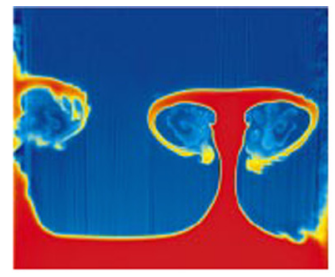

(c) $\mathrm{t}=7 \mathrm{~ms}$

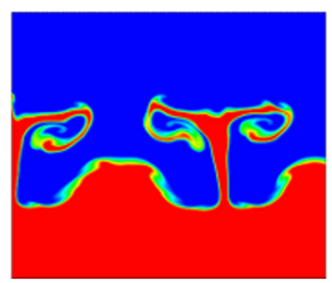

(g) $\mathrm{t}=6.63 \mathrm{~ms}$

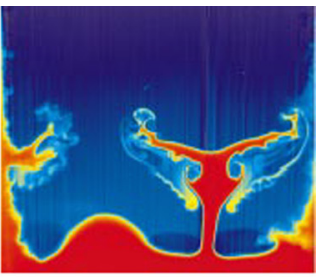

(d) $\mathrm{t}=8 \mathrm{~ms}$

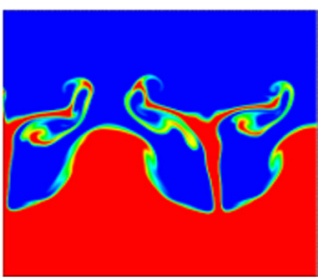

(h) t=6.84 ms

FIG. 5. Further comparison of density plots for air-SF 6 RMI (System 1) to experimental result of Collins and Jacobs [38] for times slightly before and after reshock.

amplitude to wavelength of 0.2 , which is expected to drive the instability growth immediately to the nonlinear regime. The simulation parameters during initial setup for this case are listed under System 2b in Table I. The plots of density for our simulation are shown in Fig. 9. We observe the classical mushroom-shaped structures developing at times before reshock, similar to the ones reported in [36]. Our observations show that our FCNS solver can reproduce the structures observed in RMI that are produced by a particle method such as DSMC.

\section{F. Results for system of length scale $O\left(10^{-6}\right) \mathrm{m}$}

We consider helium as the light fluid (species 1) and argon as the heavier, species 2, and a Mach 2.0 shock in helium. The system parameters are given under System 3 in Table I. The fluid-fluid interface perturbation was $\psi(y)=a_{0} \sin (k y)$, with initial perturbation amplitude $a_{0}=0.4 \mu \mathrm{m}$ and wave number $k=2 \pi / \lambda$ with wavelength $\lambda_{p}=8 \mu \mathrm{m}$. The left boundary had an inflow condition, the right wall was set to reflecting in the $\mathrm{X}$ direction, and the lower and upper $\mathrm{Y}$ walls had a reflecting condition in the $\mathrm{Y}$ direction because only half the sine wave was included in the perturbation. The results with fluctuations presented in this section are the averaged result from $N_{\text {samp }}=100$ runs. Figure 10 shows the evolution of bubble, spike, and interface location relative to the unperturbed interface. Figure 11 shows the evolution of the nondimensional amplitude of the perturbation. Figure 12 shows the plot of the growth rate of the perturbation for the nonfluctuating case and ensemble-averaged growth rate for the fluctuating simulations. We observe that the ensemble-averaged behavior and deterministic behavior match well at the linear stage and early nonlinear stage. At later stages, the ensemble-averaged behavior shows a larger growth rate and the deterministic behavior is closer to the lower bound. This is because of

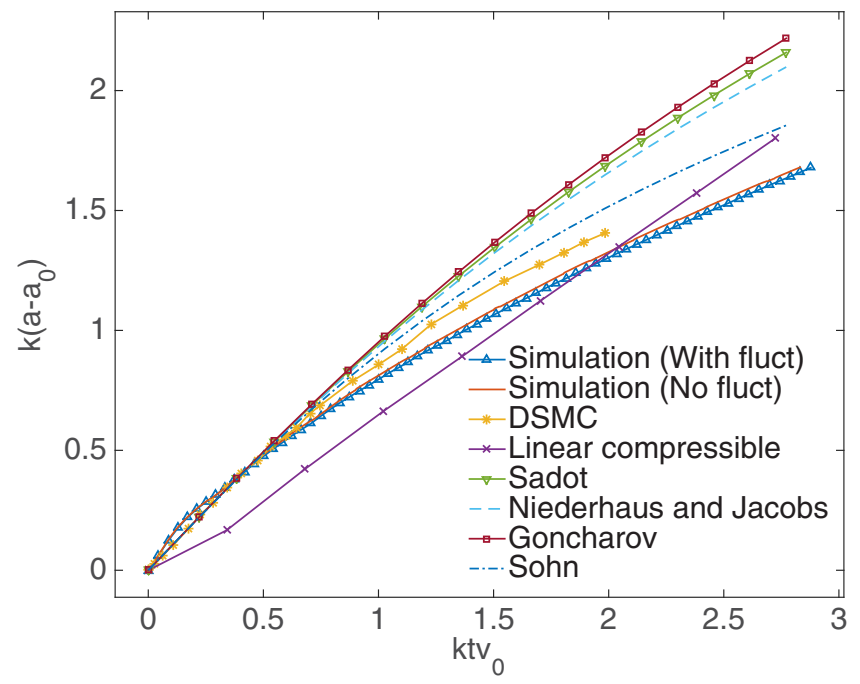

FIG. 7. Interface growth for He-Ar RMI (System 2a) at Mach 1.5, compared to DSMC result in [36]. 


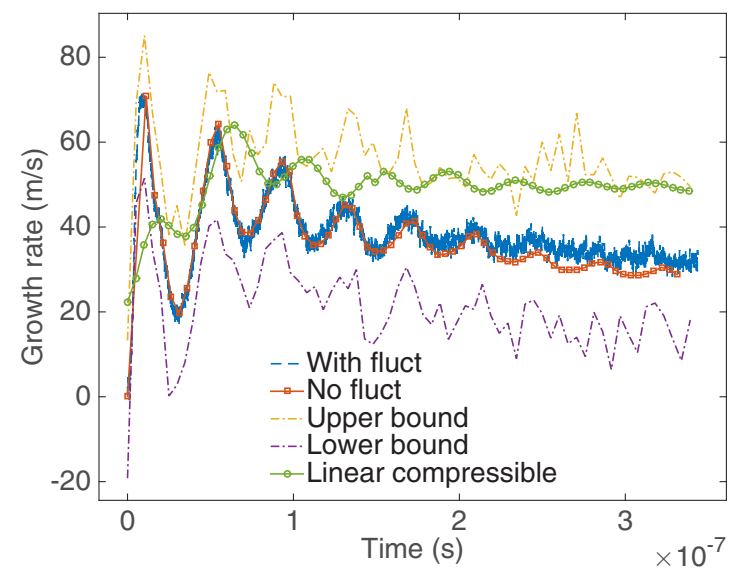

FIG. 8. Growth rate of perturbation for He-Ar RMI (System 2a) at Mach 1.5. Bounds represent extrema from among all fluctuating instances at each simulation time step.
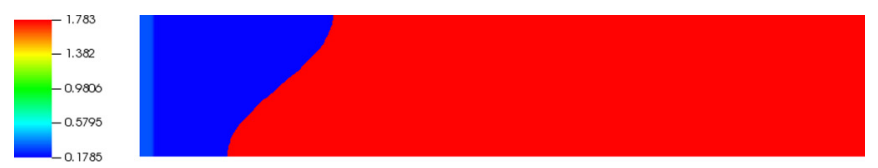

(a) $\mathrm{t}=0 \mu \mathrm{s}$

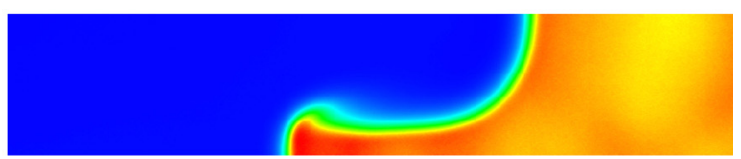

(b) $\mathrm{t}=0.2 \mu \mathrm{s}$
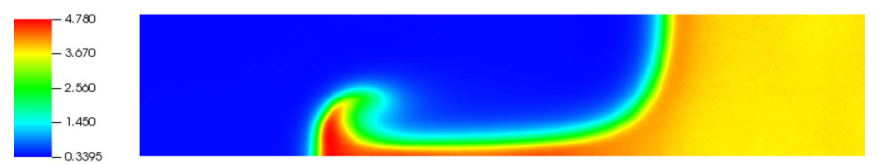

(c) $\mathrm{t}=0.4 \mu \mathrm{s}$
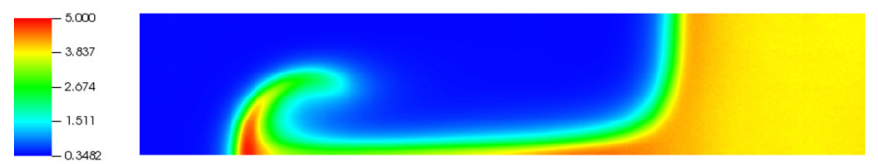

(d) $\mathrm{t}=0.6 \mu \mathrm{s}$
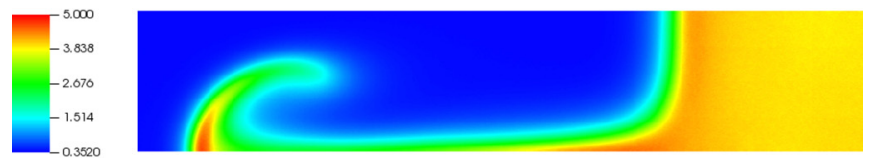

(e) $\mathrm{t}=0.7 \mu \mathrm{s}$

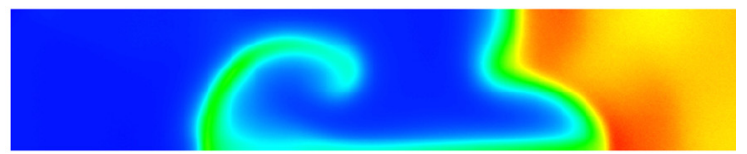

(f) $\mathrm{t}=0.9 \mu \mathrm{s}$

FIG. 9. Density plots obtained from fluctuating hydrodynamics simulation of He-Ar RMI (System 2b) at Mach 1.5 showing growth of the mixing layer with time. The perturbation-to-amplitude ratio is 0.2 . Plot shows a portion of the domain $136 \mu \mathrm{m} \times 25 \mu \mathrm{m}$. Density is in SI units.

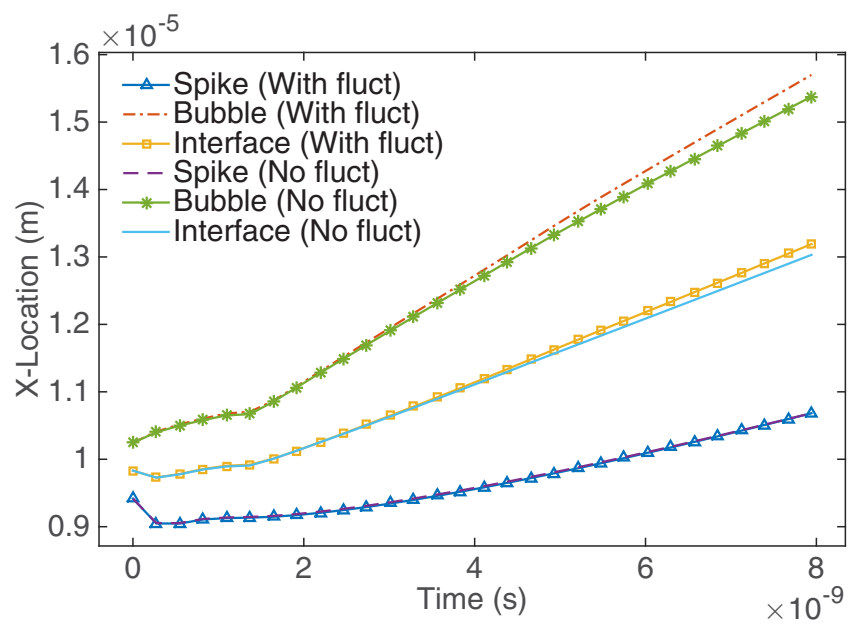

FIG. 10. Evolution of interface location for He-Ar RMI (System 3) at Mach 2.0.

the increased significance of thermal fluctuations for this system due to the Boltzmann number Bo $\sim 1$ as discussed in detail in Sec. V. Further, Figs. 10 and 11 also compare our nonlinear simulation result to the solution of the linearized compressible Euler equations using the method developed in [51]. There is some discrepancy between the two, as well as discrepancy compared to the growth models in literature. This is expected because our nonlinear simulations are performed with a diffused interface and included diffusive effects as well as the effect of thermal fluctuations.

\section{G. Results for system of length scale $O\left(10^{-7}\right) \mathrm{m}$}

We consider helium as the light fluid (species 1) and argon as the heavier, species 2, and a Mach 2.0 shock in helium. The system parameters are given under System 4 in Table I. The fluid-fluid interface perturbation was $\psi(y)=a_{0} \sin \left(\pi k y / L_{y}\right)$, with initial perturbation amplitude $a_{0}=0.0512 \mu \mathrm{m}$ and wave number $k=2 \pi / \lambda$ with wavelength $\lambda_{p}=1 \mu \mathrm{m}$. The left boundary had an inflow condition, the right wall was set to

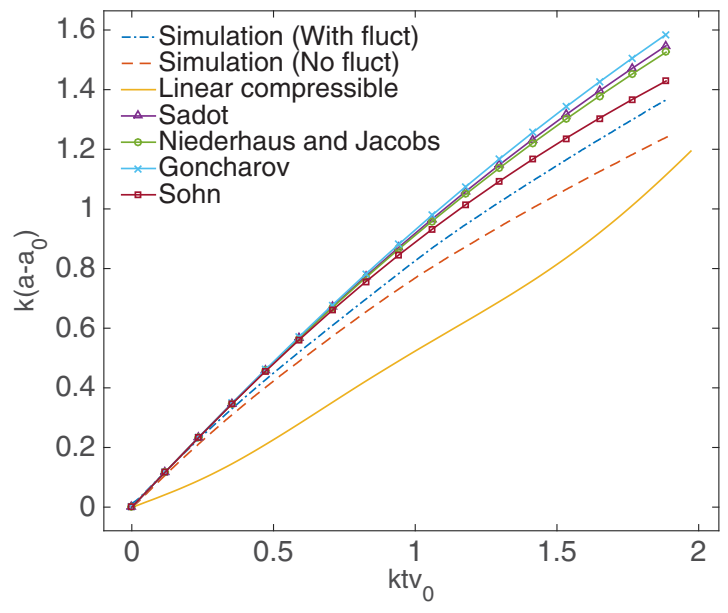

FIG. 11. Nondimensional growth rate of perturbation for $\mathrm{He}-\mathrm{Ar}$ RMI (System 3) at Mach 2.0. 


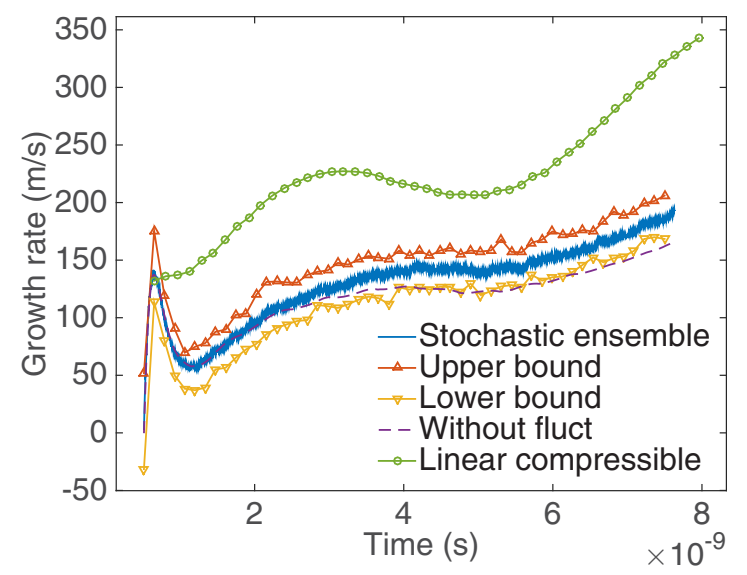

FIG. 12. Growth rate of perturbation for He-Ar RMI (System 3) at Mach 2.0. Bounds represent extrema from among all fluctuating instances at each simulation time step.

reflecting in the $\mathrm{X}$ direction, and the lower and upper $\mathrm{Y}$ walls had a reflecting condition in the $\mathrm{Y}$ direction because only half the sine wave was included in the perturbation. The results with fluctuations presented in this section are the averaged result from $N_{\text {samp }}=150$ runs, for each of which the maximum number of simulation time steps was 125000 . Figure 13 shows the evolution of bubble, spike, and interface location relative to the unperturbed interface. Figure 14 shows the evolution of the nondimensional amplitude of the perturbation. Figure 15 shows the plot of the growth rate of the perturbation for the nonfluctuating case and ensemble-averaged result for the fluctuating simulations. We see that the deterministic behavior emerges from an ensemble-averaged behavior of the fluctuating cases. Bounds on the physical system's growth rate are shown also in the figure. Owing to the inclusion of diffusive and fluctuation effects as well as a diffused interface in our nonlinear simulations, there is a discrepancy between the nonlinear simulation result and the solution of the linear compressible Euler equation, as seen from Fig. 14.

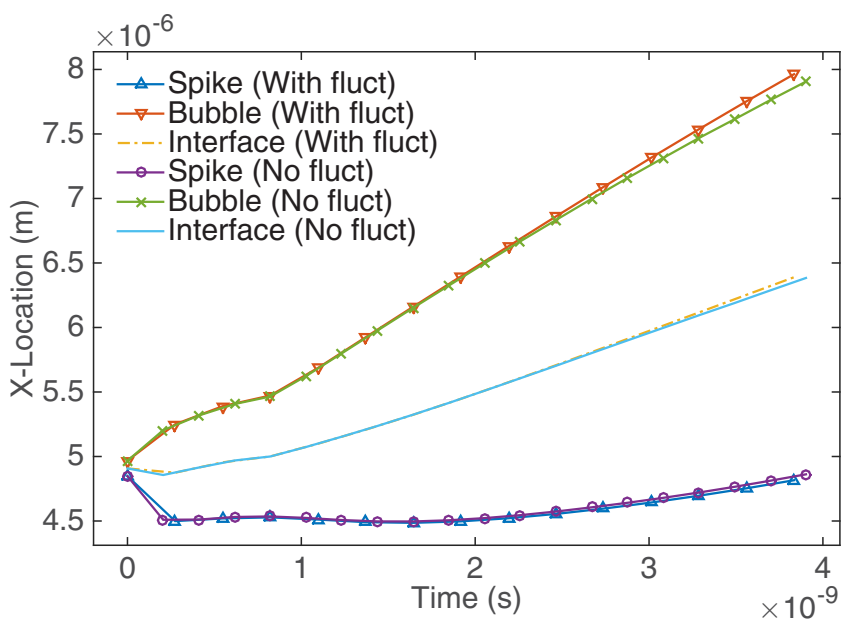

FIG. 13. Evolution of interface location for He-Ar RMI (System 4) at Mach 2.0.

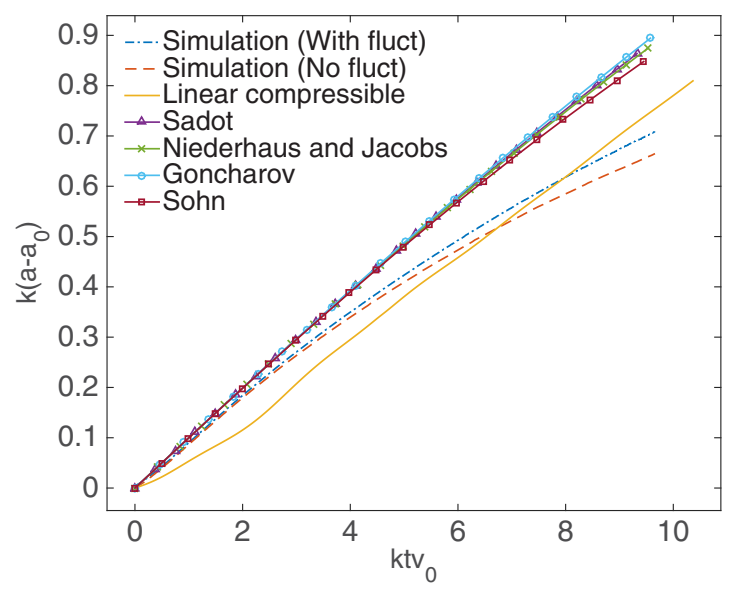

FIG. 14. Nondimensional growth rate of perturbation for $\mathrm{He}-\mathrm{Ar}$ RMI (System 4) at Mach 2.0.

\section{DISCUSSION}

In order to facilitate this discussion, we begin by defining and listing the calculated values of the nondimensional parameters associated with the three fluctuating hydrodynamic RMI systems simulated, namely, Systems 2a, 3, and 4. Nondimensionalization of the governing equations presented in Sec. II leads to five dimensionless numbers: the Reynolds number Re, Prandtl number Pr, Schmidt number Sc, Boltzmann number Bo, and Mach number Ma. Additionally here, we consider Knudsen number Kn and Péclet number Pe. Let $U_{0}, \rho_{0}$, and $L_{0}$ be the chosen characteristic velocity, reference density, and characteristic length scale, respectively. Here, we choose $\rho_{0}$ as well as transport coefficients to be values of the He-Ar gas mixture with mass fraction $Y_{1}=Y_{2}=0.5$ at STP. We choose $U_{0}$ to be the sound speed, and $L_{0}$ to be the $\mathrm{Y}$ dimension of the domain, i.e., half the perturbation wavelength. The Reynolds number measures the relative strength of convective momentum transport over diffusive momentum transport and is given by $\operatorname{Re}=\rho_{0} U_{0} L_{0} / \eta_{0}$, where $\eta_{0}$ is the reference viscosity. The Prandtl number is given by $\operatorname{Pr}=\eta_{0} C_{p} / \kappa_{0}$, where $C_{p}$ and $\kappa_{0}$ are the reference specific heat at constant pressure and thermal conductivity, respectively. The Schmidt number

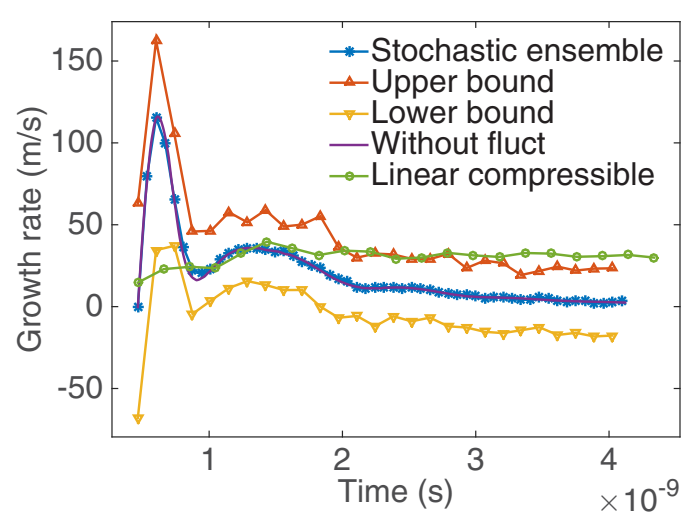

FIG. 15. Growth rate of perturbation for He-Ar RMI (System 4) at Mach 2.0. Bounds represent extrema from among all fluctuating instances at each simulation time step. 
TABLE IV. Dimensionless numbers for the fluctuating RMI systems considered. Values listed are for He-Ar mixture with species fraction $Y_{1}=Y_{2}=0.5$.

\begin{tabular}{lccc}
\hline \hline & System 2 & System 3 & System 4 \\
\hline $\operatorname{Re}$ & 1110 & 180 & 23 \\
$\mathrm{Pr}$ & 1.33 & 1.33 & 1.33 \\
$\mathrm{Sc}$ & 0.82 & 0.82 & 0.82 \\
$\mathrm{Bo}$ & 0.024 & 0.988 & 0.33 \\
$\mathrm{Kn}$ & 0.002 & 0.01 & 0.08 \\
$\mathrm{Pe}$ & 908 & 146 & 19 \\
\hline \hline
\end{tabular}

is given by $\mathrm{Sc}=\eta_{0} / \rho_{0} D_{0}$, where $D_{0}$ is the reference diffusion coefficient. The Boltzmann number is given by

$$
\mathrm{Bo}=\sqrt{\frac{2}{3}} \frac{v_{t h}}{U_{0}} \sqrt{\frac{\alpha}{n_{0}}},
$$

where $v_{t h}=\sqrt{3 k_{B} T_{0} / m_{0}}$ is the thermal velocity at reference temperature $T_{0}, \alpha=\frac{L_{0}}{h_{0}}$, and $n_{0}=\rho_{0} h_{0}{ }^{3} / m_{0}$ is the number of molecules with molecular mass $m_{0}$ contained in the volume $h_{0}{ }^{3}$. Bo determines the relative magnitude of thermal fluctuations. Our definition of the Boltzmann number is consistent with the definition in [53], where the authors define the Boltzmann number for velocity fluctuations as $\mathrm{Bo}=$ $\left(\left\langle u^{2}\right\rangle-\langle u\rangle^{2}\right)^{1 / 2} /\langle u\rangle=(1 /\langle u\rangle) \sqrt{k_{B} T_{0} / \rho\left(L_{0}\right)^{3}}$, where the second equality uses the mean square velocity of a volume of fluid $\left(L_{0}\right)^{3}$ at thermal equilibrium. The Knudsen number measures rarefaction effects and is given by $\mathrm{Kn}=\lambda / L$, where $\lambda$ is the mean free path. The Péclet number measures the relative strength of convective mass transfer over diffusive mass transfer and is given by $\mathrm{Pe}=U_{0} L_{0} / D_{0}$. Table IV lists the dimensionless numbers for the three fluctuating RMI systems simulated. Systems 2a, 3, and 4 are of decreasing order of magnitude of the length scale. As we decrease the length, Reynolds and Péclet numbers decrease and Knudsen number increases in magnitude. The FCNS model is a coarse-grained model that captures hydrodynamic fluctuations and is therefore valid at high $\mathrm{Re}$, high Pe, and low Kn. The systems considered satisfy these criteria. In Table IV, the Knudsen number for the post-shocked gas mixture is listed. The values for all three systems are much less than unity. We note, however, that the Knudsen number of the pre-shock gases was much less than unity for Systems 2a and 3, and was $O\left(10^{-1}\right)$ for System 4. Admittedly, for System 4 we are pushing the limits of applicability of the continuum hypothesis. From the expressions for the nondimensional parameters $\mathrm{Re}, \mathrm{Pr}, \mathrm{Sc}$, and Bo, it can be concluded that Bo can be changed independent of the other three provided $\rho_{0}, \rho_{0} L_{0}, L_{0}$, and the transport coefficients are held constant. The significance of thermal fluctuations is therefore determined by the Boltzmann number as defined in Eq. (20). Thermal fluctuations are insignificant if Bo $\ll 1$ and are of increasing significance as Bo gets closer to 1 . From Table IV, we see that Bo for System 3 is close to 1 , which explains the departure from deterministic mixing behavior at larger times that is seen from Fig. 12 .

\section{CONCLUSIONS}

Thermal fluctuations play a key role in two-fluid instabilities and the mixing behavior thereof; they trigger the onset of linear growth of the perturbation, thereby affecting the flow [1], they provide nucleation sites for the breakup of large flow features at late stages of growth [24], and the deterministic flow field can emerge as the ensemble-averaged solution of many fluctuating instances even for systems as small as $1 \mu \mathrm{m}$ [28]. Here, we use numerical solutions of the two-fluid FCNS equations to study the effect of these fluctuations on the mixing behavior for the RMI. The numerical method used was successfully verified in two stages: for the deterministic fluxes by comparison against the results of the air- $\mathrm{SF}_{6}$ RMI experiment reported in [38], and for the stochastic terms by comparison against the direct simulation Monte Carlo results for He-Ar RMI in [36]. In total, we performed fluctuating hydrodynamic RMI simulations for three He-Ar systems of length scales that differed by an order of magnitude that was determined by the domain length; a system identical to the one in [36] of length scale $O\left(10^{-5}\right) \mathrm{m}$ that also constituted the aforementioned stochastic verification case, and two other systems of length scales $O\left(10^{-6}\right) \mathrm{m}$ and $O\left(10^{-7}\right) \mathrm{m}$, respectively. By studying systems of length scales of decreasing order of magnitude, we were able to investigate a range of length scales that spanned between macroscopic and mesoscopic with varying levels of fluctuations characterized by the nondimensional Boltzmann number (Bo). In doing so, we also suggested a regularization for the spatiotemporal discretization of the noise terms in the governing equations. Traditionally, as explained in [40], a space-time white noise field $Z(x, t)$ is discretized for spatial interval $h$ and time interval $\Delta t$ as $Z(x, t) \rightarrow 1 / \sqrt{h \Delta t} N(i h, n \Delta t)$, where $N$ is a Gaussian random field of mean zero and variance 1 , and the factor $1 / \sqrt{h \Delta t}$ is the regularization. For a multidimensional FCNS system on a regular Cartesian grid, when using such a regularization, the spatial discretization of the stochastic terms should be such that the cell width $h \geqslant h_{0}$, where $h_{0}$ corresponds to a cell volume that contains enough molecules of the fluid such that the fluctuations are physically meaningful in that they produce the right equilibrium spectrum as mentioned in [29,31]. For the RMI systems of length scale $O\left(10^{-6}\right) \mathrm{m}$ and $O\left(10^{-7}\right) \mathrm{m}$, it was desired to use a cell size smaller than this limit in order to resolve the viscous shock. This was achieved by using a modified regularization via $Z(x, t) \rightarrow$ $1 / \sqrt{\Delta t \max \left(h^{3}, h_{0}^{3}\right)} N(i h, n \Delta t)$, with $h_{0}=\xi h \forall h<h_{0}, \xi \in$ $\mathbb{Z}^{+}$and where $h_{0}$ was determined for the two-fluid mixture by a successful verification of the equilibrium static structure factors it produced. Our simulations show that for systems with Bo $\ll 1$ deterministic mixing behavior emerges as the ensemble-averaged behavior of several fluctuating instances, whereas when $\mathrm{Bo} \approx 1$, a deviation from deterministic behavior is observed. For all cases, the FCNS solution provides bounds on the growth rate of the amplitude of the mixing layer.

\section{ACKNOWLEDGMENTS}

We thank the KAUST Supercomputing Lab for use of the Cray XC-40, Shaheen II. This work was funded by the KAUST baseline research funds. 


\section{APPENDIX A: NUMERICAL METHOD}

\section{Discretization of underlying space}

The underlying space is discretized using the finite volume method into a Cartesian mesh of grid points $\left(\mathbf{i}_{1}, \ldots, \mathbf{i}_{D}\right)=$ $\mathbf{i} \in \mathrm{Z}^{\mathrm{D}}$. The grid points are the centers of rectangular control volumes, also called cells. The cell-centered discretization of the problem domain $\Omega$ into a collection of control volumes is denoted by $\Gamma \subset \mathrm{Z}^{\mathrm{D}}$. Therefore, $\Gamma$ is the discrete set that indexes the cell centers and is in general also used to index the cells themselves. If $\mathbf{x}_{0} \in \mathbb{R}^{\mathrm{D}}$ is a fixed origin, $h$ the mesh spacing, and $\mathbf{v}$ the vector whose entries are all 1 , then $\mathbf{i} \in \Gamma$ represents a control volume, i.e., a region of space $\left[\mathbf{x}_{0}+\left(\mathbf{i}-\frac{1}{2} \mathbf{v}\right) h, \mathbf{x}_{0}+\left(\mathbf{i}+\frac{1}{2} \mathbf{v}\right) h\right]$. The volume of each of the cells is given by the intersection of this control volume with the physical domain $\Omega$, i.e., $V_{\mathbf{i}}=$ $\left[\mathbf{x}_{0}+\left(\mathbf{i}-\frac{1}{2} \mathbf{v}\right) h, \mathbf{x}_{0}+\left(\mathbf{i}+\frac{1}{2} \mathbf{v}\right) h\right] \bigcap \Omega$. We also have edgecentered discretization $\Gamma^{\mathrm{e}^{d}}$, i.e., a set of points of the form $\left[\mathbf{x}_{0}+\left(\mathbf{i} \pm \frac{1}{2} \mathbf{e}^{d}\right) h, \mathbf{i} \in \Gamma\right]$, where $\mathbf{e}^{d}$ is the unit vector in the $d$ direction. Then, $\Gamma^{\mathbf{e}^{d}}$ is the discrete set that indexes the faces of the cells in $\Gamma$ whose normals are $\mathbf{e}^{d}$. The intersection of the boundary of each control volume with coordinate planes $\left\{\mathbf{x}: x_{d}=x_{0 d}+\left(i_{d} \pm \frac{1}{2}\right) h\right\}$ is denoted as $A_{\mathbf{i} \pm \mathbf{e}^{d} / 2}$, is given by $A_{\mathbf{i} \pm \mathbf{e}^{d} / 2}=\left[\left(\mathbf{i}+\mathbf{e}^{d}\right) h,(\mathbf{i}+\mathbf{v}) h\right], \mathbf{i}+\frac{1}{2} \mathbf{e}^{d} \in \Gamma^{\mathbf{e}^{d}}$. The centroids of the faces of the cells are given by

$$
x_{\mathbf{i} \pm \mathbf{e}^{d} / 2}=\left(\frac{1}{\left|A_{\mathbf{i} \pm \mathbf{e}^{d} / 2}\right|} \int_{A_{\mathbf{i} \pm \mathbf{e}^{d} / 2}} \mathbf{x} d A\right)-\left(\mathbf{i} \pm \frac{1}{2} \mathbf{e}^{d}\right) h .
$$

A cell-centered discrete variable $\phi$ on $\Gamma$ is defined as $\phi$ : $\Gamma \rightarrow \mathbb{R}^{m}$, and the value of $\phi$ at cell $\mathbf{i} \in \Gamma$ is denoted by $\phi_{\mathbf{i}} \in$ $\mathbb{R}^{m} \cdot \rho, \mathbf{u}, P$, and the conserved densities of mass $\rho$, linear momentum $\rho \mathbf{u}$, energy $E$, and species 1 mass $\rho Y_{1}$ are all cellcentered quantities and constitute the discrete solution variable $\mathbf{U}$. The noise $\mathbf{N}$ is also discretized in space as a cell-centered quantity. We also define face-centered vector fields such as the fluxes, on $\Gamma^{\mathbf{e}^{d}}$. We define $|\mathbf{i}|=\max _{d=1, \ldots, D}\left(\left|i_{d}\right|\right)$ and the set of all points within a $|\cdot|$ distance $p$ of $\Gamma$, i.e., the ghost mesh, as $\mathcal{G}(\Gamma, p)=\bigcup_{|\mathbf{i}| \leqslant p} \Gamma+\mathbf{i}$.

\section{Discretized conservation form of the governing equations}

We rewrite Eq. (1) as

$$
\frac{\partial \mathcal{U}}{\partial t}=-\sum_{d=1}^{D} \frac{\partial \mathcal{F}^{d}}{\partial x_{d}}
$$

where

$$
\mathcal{F}=\mathcal{F}_{H}-\mathcal{F}_{G}-\mathcal{F}_{Z}
$$

The underlying space is discretized such that the solution $\mathbf{U}$ is defined at the cell centers and the discrete fluxes $\mathbf{F}_{H}$, $\mathbf{F}_{G}$, and $\mathbf{F}_{Z}$ are defined at the face centers. We discretize the solution to Eq. (A2) in time and space, approximating $\mathcal{U}$ by the values of cell centers of the Cartesian grid, i.e., $\mathcal{U}=\mathbf{U}_{\mathbf{i}}^{n} \approx \mathrm{U}\left(\mathbf{i} h, n \Delta t, \mathbf{N}_{i}^{n}\right), \mathbf{i} \in Z^{\mathrm{D}}$, where $\mathbf{N}_{\mathbf{i}}^{n}$ is the spatiotemporal discretization of the noise $\mathbf{N} \equiv \mathbf{N}(\mathbf{x}, t)$. The conservative discretization of Eq. (A2) is obtained by integrating it over the control volume $V_{\mathbf{i}}$ of cell $\mathbf{i}$ as

$$
\frac{d}{d t} \int_{A_{\mathbf{i}+\mathbf{e}^{d} / 2}}^{A_{\mathbf{i}-\mathbf{e}^{d} / 2}} \mathbf{U} d A=-\sum_{d=1}^{D} \int_{A_{\mathbf{i}+\mathbf{e}^{d} / 2}}^{A_{\mathbf{i}-\mathbf{e}^{d} / 2}} \frac{\partial \overrightarrow{\mathcal{F}}^{d}}{\partial x_{d}} d A .
$$

We define

$$
\mathbf{U}_{\mathrm{tot}, \mathbf{i}}:=\int_{A_{\mathbf{i}+\mathbf{e}^{d} / 2}}^{A_{\mathbf{i}-\mathbf{e}^{d} / 2}} \mathbf{U} d A=\int_{V_{\mathbf{i}}} \mathbf{U} d V,
$$

and the cell-averaged solution as

$$
\langle\mathbf{U}\rangle_{\mathbf{i}}:=\frac{1}{V_{\mathbf{i}}} \mathbf{U}_{t o t, \mathbf{i}}=\frac{1}{h^{D}} \int_{V_{\mathbf{i}}} \mathbf{U} d V .
$$

Using Eq. (A6) in Eq. (A4) gives the evolution of the spatially discretized governing equations via a method-of-lines approach as

$$
\frac{d\langle\mathbf{U}\rangle_{\mathbf{i}}}{d t}=-\frac{1}{h^{D}} \int_{V_{\mathbf{i}}} \nabla \cdot \overrightarrow{\mathcal{F}} d V \approx-(D \cdot \overrightarrow{\mathcal{F}})_{\mathbf{i}},
$$

where the discretized divergence operator $(D \cdot \overrightarrow{\mathcal{F}})_{\mathbf{i}}$ is given by the quadrature rule

$$
(D \cdot \overrightarrow{\mathcal{F}})_{\mathbf{i}}=\frac{1}{h}\left[\sum_{d=1}^{D}\left(\left\langle\overrightarrow{\mathcal{F}}^{d}\right\rangle_{\mathbf{i}+\frac{1}{2} \mathbf{e}^{d}}-\left\langle\overrightarrow{\mathcal{F}}^{d}\right\rangle_{\mathbf{i}-\frac{1}{2} \mathbf{e}^{d}}\right)\right],
$$

and the face-centered fluxes are

$$
\left\langle\mathcal{F}^{d}\right\rangle_{\mathbf{i}+\frac{1}{2} \mathbf{e}^{d}}=\frac{1}{h^{D-1}} \int_{A_{\mathbf{i}+\mathbf{e}^{d} / 2}}^{A_{\mathbf{i}-\mathbf{e}^{d} / 2}} \overrightarrow{\mathcal{F}}^{d} d A .
$$

Substituting Eq. (A8) in Eq. (A7) we get the final spatially discretized governing equations that are decoupled from the temporal discretization as

$$
\frac{d\langle\mathbf{U}\rangle_{\mathbf{i}}}{d t}=-\frac{1}{h}\left[\sum_{d=1}^{D}\left(\left\langle\overrightarrow{\mathcal{F}}^{d}\right\rangle_{\mathbf{i}+\frac{1}{2} \mathbf{e}^{d}}-\left\langle\overrightarrow{\mathcal{F}}^{d}\right\rangle_{\mathbf{i}-\frac{1}{2}} \mathbf{e}^{d}\right)\right] .
$$

\section{Numerical fluxes}

In order to numerically solve Eq. (A10), the fluxes at the face centers are required at each time step. Calculation of the fluxes at the face centers requires the transformation from cell centers to face centers. We resort to the numerical estimation to the discretized fluxes at the face centers, by approximating $(D \cdot \overrightarrow{\mathcal{F}})_{\mathbf{i}}$ as

$$
(D \cdot \overrightarrow{\mathcal{F}})_{\mathbf{i}} \approx\left[D^{F}(\mathbf{U})\right]_{\mathbf{i}}-\left[D^{G}(\mathbf{U})\right]_{\mathbf{i}}-\left[D^{Z}(\mathbf{U})\right]_{\mathbf{i}},
$$

where $\left[D^{F}(\mathbf{U})\right]_{\mathbf{i}},\left[D^{G}(\mathbf{U})\right]_{\mathbf{i}}$, and $\left[D^{Z}(\mathbf{U})\right]_{\mathbf{i}}$ are the numerical approximations to the hyperbolic, viscous, and stochastic parts of the divergence of flux $\mathcal{F}$. $\left[D^{F}(\mathbf{U})\right]_{\mathbf{i}}$ is given by

$$
\left[D^{F}(\mathbf{U})\right]_{\mathbf{i}}=\frac{1}{h} \sum_{d=1}^{D} \hat{F}_{H, \mathbf{i}+\frac{1}{2} \mathbf{e}^{d}}^{d}-\hat{F}_{H, \mathbf{i}-\frac{1}{2} \mathbf{e}^{d}}^{d}
$$


where the fluxes denoted with a ${ }^{\wedge}$ on top are the numerical fluxes at the face centers. $D^{G}(\mathbf{U})_{\mathbf{i}}$ is given by

$$
\begin{aligned}
{\left[D^{G}(\mathbf{U})\right]_{\mathbf{i}} } & =\frac{1}{h} \sum_{d=1}^{D} \hat{G}_{\mathbf{i}+\frac{1}{2} \mathbf{e}^{d}}^{d}-\hat{G}_{\mathbf{i}-\frac{1}{2} \mathbf{e}^{d}}^{d} \\
& :=\left(\begin{array}{c}
0 \\
{\left[L^{\tau}(\mathbf{u})\right]_{\mathbf{i}}} \\
{\left[L^{d}(\mathbf{u})\right]_{\mathbf{i}}-\left[L^{q}(\mathbf{q})\right]_{\mathbf{i}}} \\
{\left[L^{f}\left(\mathbf{f}_{1}\right)\right]_{\mathbf{i}}}
\end{array}\right),
\end{aligned}
$$

where $\left[L^{\tau}(\mathbf{u})\right]_{\mathbf{i}},\left[L^{q}(\mathbf{q})\right]_{\mathbf{i}},\left[L^{d}(\mathbf{u})\right]_{\mathbf{i}}$, and $\left[L^{f}\left(\mathbf{f}_{1}\right)\right]_{\mathbf{i}}$ are discretizations of $\boldsymbol{\nabla} \cdot \boldsymbol{\tau}, \boldsymbol{\nabla} \cdot \mathbf{q}, \boldsymbol{\nabla} \cdot(\boldsymbol{\tau} \cdot \mathbf{u})$, and $\boldsymbol{\nabla} \cdot \mathbf{f}_{1}$, respectively. $\left[D^{Z}(\mathbf{U})\right]_{\mathbf{i}}$ is defined as

$$
\begin{aligned}
{\left[D^{Z}(\mathbf{U})\right]_{\mathbf{i}} } & =\frac{1}{h} \sum_{d=1}^{D} \hat{Z}_{\mathbf{i}+\frac{1}{2} \mathbf{e}^{d}}^{d}-\hat{Z}_{\mathbf{i}-\frac{1}{2} \mathbf{e}^{d}}^{d} \\
& :=\left(\begin{array}{c}
0 \\
{\left[L^{z \tau}(\tilde{\boldsymbol{\tau}})\right]_{\mathbf{i}}} \\
{\left[L^{z d}(\tilde{\boldsymbol{\tau}}, \tilde{\mathbf{q}})\right]_{\mathbf{i}}+\left[L^{z q}(\tilde{\mathbf{q}})\right]_{\mathbf{i}}} \\
{\left[L^{z f}\left(\tilde{\mathbf{f}}_{1}\right)\right]_{\mathbf{i}}}
\end{array}\right),
\end{aligned}
$$

where $\left[L^{z \tau}(\tilde{\boldsymbol{\tau}})\right]_{\mathbf{i}},\left[L^{z q}(\tilde{\mathbf{q}})\right]_{\mathbf{i}},\left[L^{z d}(\tilde{\boldsymbol{\tau}}, \tilde{\mathbf{q}})\right]_{\mathbf{i}}$, and $\left[L^{z f}\left(\tilde{\mathbf{f}}_{1}\right)\right]_{\mathbf{i}}$ are discretizations of $(\nabla \cdot \tilde{\boldsymbol{\tau}}),(\nabla \cdot \tilde{\boldsymbol{q}}), \boldsymbol{\nabla} \cdot(\tilde{\boldsymbol{\tau}} \cdot \tilde{\mathbf{u}})$, and $\boldsymbol{\nabla} \cdot \tilde{\mathbf{f}}_{1}$. The goal of every numerical method is to approximate the fluxes as best as possible using numerical fluxes.

\section{a. Explicit method for the deterministic numerical fluxes $\left[D^{F}(\mathbf{U})\right]_{\mathrm{i}}$ and $\left[D^{G}(\mathbf{U})\right]_{\mathrm{i}}$}

A transformation $\mathbf{U}$ to primitive $\mathbf{W}(\mathbf{U})$ is carried out. For the hyperbolic part, dropping the ^ on the numerical fluxes for brevity, we have that the face-averaged flux in the $d$ dimension on the high face, i.e., $\left\langle F_{H}^{d}\right\rangle_{\mathbf{i}+\frac{1}{2} \mathrm{e}^{d}}$, is given by

$$
\left\langle F_{H}^{d}\right\rangle_{\mathbf{i}+\frac{1}{2} \mathbf{e}^{d}}=F_{H}^{d}\left(W_{\mathbf{i}+\frac{1}{2} \mathbf{e}^{d}}^{d}\right)=F_{H}^{d}\left(R_{P}\left\{W_{\mathbf{i}+\frac{1}{2} \mathbf{e}^{d}}^{d}, W_{\mathbf{i}-\frac{1}{2} \mathbf{e}^{d}}^{d}\right\}\right),
$$

where $R_{P}\left\{W_{\mathbf{i}+\frac{1}{2} \mathbf{e}^{d}}^{d}, W_{\mathbf{i}-\frac{1}{2} \mathbf{e}^{d}}^{d}\right\}$ denotes the solution to the Riemann problem at the cell edges. This constitutes a Godunov method that is second order accurate in the absence of stochasticity. For the viscous numerical flux, we do the following. We have

$$
\left[L^{\tau}(\mathbf{u})\right]_{\mathbf{i}}=\frac{1}{h} \sum_{d=1}^{D}\left(\boldsymbol{\tau}_{\mathbf{i}+\frac{1}{2} \mathbf{e}^{d}}-\boldsymbol{\tau}_{\mathbf{i}-\frac{1}{2} \mathbf{e}^{d}}\right),
$$

where the face-centered shear stress is calculated as

$$
\boldsymbol{\tau}_{\mathbf{i}+\frac{1}{2} \mathbf{e}^{d}}=\frac{1}{2}\left(\boldsymbol{\tau}_{\mathbf{i}+1}+\boldsymbol{\tau}_{\mathbf{i}}\right)
$$

where the cell-centered shear stress is calculated as

$$
\boldsymbol{\tau}_{\mathbf{i}}=2 \eta_{\mathbf{i}}(\nabla \mathbf{u})_{\mathbf{i}}-\frac{2}{3} \eta_{\mathbf{i}}(\nabla \cdot \mathbf{u})_{\mathbf{i}} \mathbf{I},
$$

and where the gradient of the velocity at the cell center is calculated as

$$
(\nabla \mathbf{u})_{\mathbf{i}}=\frac{1}{h}\left(\frac{1}{2}\left(\mathbf{u}_{i+1}-\mathbf{u}_{i-1}\right)\right) .
$$

For the divergence of heat flux we have

$$
\left[L^{q}(\mathbf{q})\right]_{\mathbf{i}}=\frac{1}{h} \sum_{d=1}^{D}\left(\kappa_{\mathbf{i}+\frac{1}{2} \mathbf{e}^{d}}(\nabla T)_{\mathbf{i}+\frac{1}{2} \mathbf{e}^{d}}-\kappa_{\mathbf{i}-\frac{1}{2} \mathbf{e}^{d}}(\nabla T)_{\mathbf{i}-\frac{1}{2} \mathbf{e}^{d}}\right),
$$

where

$$
\kappa_{\mathbf{i}+\frac{1}{2} \mathbf{e}^{d}}(\nabla T)_{\mathbf{i}+\frac{1}{2} \mathbf{e}^{d}}=\frac{1}{2}\left(\kappa_{\mathbf{i}+1}(\nabla T)_{\mathbf{i}+1}+\kappa_{\mathbf{i}}(\nabla T)_{\mathbf{i}}\right),
$$

where

$$
(\nabla T)_{\mathbf{i}}=\frac{1}{h}\left(\frac{1}{2}\left(T_{\mathbf{i}+1}-T_{\mathbf{i}-1}\right)\right) .
$$

For the divergence of the viscous heating term we have

$$
\left[L^{d}(\mathbf{u})\right]_{\mathbf{i}}=\frac{1}{h} \sum_{d=1}^{D}\left(\boldsymbol{\tau}_{\mathbf{i}+\frac{1}{2} \mathbf{e}^{d}} \cdot \mathbf{u}_{\mathbf{i}+\frac{1}{2} \mathbf{e}^{d}}-\boldsymbol{\tau}_{\mathbf{i}-\frac{1}{2} \mathbf{e}^{d}} \cdot \mathbf{u}_{\mathbf{i}+\frac{1}{2} \mathbf{e}^{d}}\right) .
$$

For the divergence of species diffusion flux, the stencil for $\left[L^{f}\left(\mathbf{f}_{1}\right)\right]_{\mathbf{i}}$ is similar to that of $\left[L^{q}(\mathbf{q})\right]_{\mathbf{i}}$.

\section{b. Explicit method for the stochastic numerical flux $D^{Z}(\mathrm{U})_{\mathrm{i}}$}

The divergence of the stochastic stress tensor is calculated as

$$
\left[L^{z \tau}(\tilde{\boldsymbol{\tau}})\right]_{\mathbf{i}}=\frac{1}{h} \sum_{d=1}^{D}\left(\tilde{\boldsymbol{\tau}}_{\mathbf{i}+\frac{1}{2} \mathbf{e}^{d}}-\tilde{\boldsymbol{\tau}}_{\mathbf{i}-\frac{1}{2} \mathbf{e}^{d}}\right),
$$

where the stochastic stress at the face center is calculated as

$$
\tilde{\boldsymbol{\tau}}_{\mathbf{i}+\frac{1}{2} \mathbf{e}^{d}}=\frac{1}{2}\left(\mathcal{S}_{\mathbf{i}+1}+\mathcal{S}_{\mathbf{i}}\right),
$$

and where the cell-centered stochastic stress in two dimensions is calculated as

$$
\tilde{\boldsymbol{\tau}}_{\mathbf{i}}=2 \sqrt{k_{B} T_{\mathbf{i}}}\left(\sqrt{\eta_{\mathbf{i}}}\right) \Re \tilde{\mathbf{N}}_{\mathbf{i}}^{s},
$$

where the dilatational contribution has been ignored because $\zeta$ is zero for a perfect gas and where the regularization factor is $\mathfrak{R}=1 / \sqrt{\Delta t \max \left(h^{3}, h_{0}^{3}\right)}$. $\mathbf{N}$ is a random Gaussian matrix with zero mean and covariance given by $\left\langle N_{p q}^{s} N_{r s}^{s}\right\rangle=\delta_{p q} \delta_{r s}$, and $\overline{\mathbf{N}}^{s}$ is the traceless symmetric part of $\mathbf{N}^{s}$, which at each cell is given by

$$
\overline{\mathbf{N}}_{\mathbf{i}}^{s}=\frac{\mathbf{N}_{\mathbf{i}}^{s}+\mathbf{N}_{\mathbf{i}}^{s T}}{2}-\frac{\operatorname{tr}\left(\mathbf{N}_{\mathbf{i}}^{s}\right)}{3} \mathbf{I} .
$$

The divergence of the stochastic heat flux is calculated as

$$
\left[L^{z q}(\tilde{\mathbf{q}})\right]_{\mathbf{i}}=\frac{1}{h} \sum_{d=1}^{D}\left(\tilde{\mathbf{q}}_{\mathbf{i}+\frac{1}{2} \mathbf{e}^{d}}-\tilde{\mathbf{q}}_{\mathbf{i}-\frac{1}{2} \mathbf{e}^{d}}\right),
$$

where the face-centered stochastic heat flux is calculated as

$$
\tilde{\mathbf{q}}_{\mathbf{i}+\frac{1}{2} \mathbf{e}^{d}}=\frac{1}{2}\left(\tilde{\mathbf{q}}_{\mathbf{i}+1}+\tilde{\mathbf{q}}_{\mathbf{i}}\right),
$$

where the cell-centered stochastic heat flux for $d=2$ is calculated as

$$
\tilde{\mathbf{q}}_{\mathbf{i}}=T_{\mathbf{i}} \sqrt{2 k_{B} \kappa_{\mathbf{i}}} \Re \mathbf{N}_{\mathbf{i}}^{q},
$$

and where $\mathbf{N}_{\mathbf{i}}^{q}$ is a random Gaussian vector of zero mean and covariance given by $\left\langle N_{m}^{q} N_{n}^{q}\right\rangle=\delta_{m n}$. The divergence of the 
stochastic species diffusion flux is calculated as

$$
\left[L^{z f}\left(\tilde{\mathbf{f}}_{1}\right)\right]_{\mathbf{i}}=\frac{1}{h} \sum_{d=1}^{D}\left(\tilde{\mathbf{f}}_{1_{\mathbf{i}+\frac{1}{2}} \mathbf{e}^{d}}-\tilde{\mathbf{f}}_{1_{\mathbf{i}-\frac{1}{2}} \mathbf{e}^{d}}\right),
$$

with

$$
\begin{gathered}
\tilde{\mathbf{f}}_{\mathbf{i}+\frac{1}{2} \mathbf{e}^{d}}=\frac{1}{2}\left(\tilde{\mathbf{f}}_{\mathbf{i}+1}+\tilde{\mathbf{f}_{\mathbf{1}}}\right), \\
\tilde{\mathbf{f}_{1 \mathbf{i}}}=\sqrt{2 D \rho_{\mathbf{i}}\left[Y_{1} Y_{2}\left(w_{1} Y_{1}+w_{2} Y_{2}\right)\right]_{\mathbf{i}}} \Re \mathbf{N}_{\mathbf{i}}^{Y_{1}},
\end{gathered}
$$$$
\text { and }\left\langle N_{m}^{Y_{1}} N_{n}^{Y_{1}}\right\rangle=\delta_{m n} \text {. }
$$

\section{Time integration}

The system of equations that are integrated in time in the absence of external body force is

$$
\begin{aligned}
\frac{d\langle\mathbf{U}\rangle_{\mathbf{i}}}{d t} & =-\left\{\left[D^{F}(\mathbf{U})\right]_{\mathbf{i}}-\left[D^{G}(\mathbf{U})\right]_{\mathbf{i}}-\left[D^{Z}\left(\mathbf{U}, \mathbf{N}^{s}, \mathbf{N}^{q}, \mathbf{N}^{Y_{1}}\right)\right]_{\mathbf{i}}\right\} \\
& \equiv \mathbf{R}\left(\mathbf{U}_{\mathbf{i}}, \mathbf{N}^{R N G}\right),
\end{aligned}
$$

where $\mathbf{N}^{R N G}=\left(\mathbf{N}^{s}, \mathbf{N}^{q}, \mathbf{N}^{Y_{1}}\right)$. For time integration, we first tested the stochastic extension to the third order Runge Kutta (SRK3) numerical scheme. For the deterministic compressible Navier-Stokes equations, the RK3 scheme is known to be a total variation diminishing (TVD) scheme, which is stable in the inviscid limit. Here, the scheme is extended for the case of stochastic fluxes by using the weights suggested in [54]. This scheme is discussed in detail in [31,54], where it was shown that, for the weights suggested, the time integration was weakly second order accurate for the case of additive noise. We observed that for the small system sizes considered in Secs. IV F and IV G, the time step was determined by viscous stability limit and that in a nonequilibrium setting like the RMI problem, no significant gains were to be achieved by using this scheme as compared to the Euler-Maruyama scheme, which uses fewer random numbers per time step. We therefore used the Euler-Maruyama scheme for all the results presented in this paper. This scheme can be summarized as

$$
\langle\mathbf{U}\rangle_{\mathbf{i}}^{n+1}=\langle\mathbf{U}\rangle_{\mathbf{i}}^{n}+(\Delta t) \mathbf{R}\left(\langle\mathbf{U}\rangle_{\mathbf{i}}^{n}, \mathbf{N}^{R N G}\right) .
$$

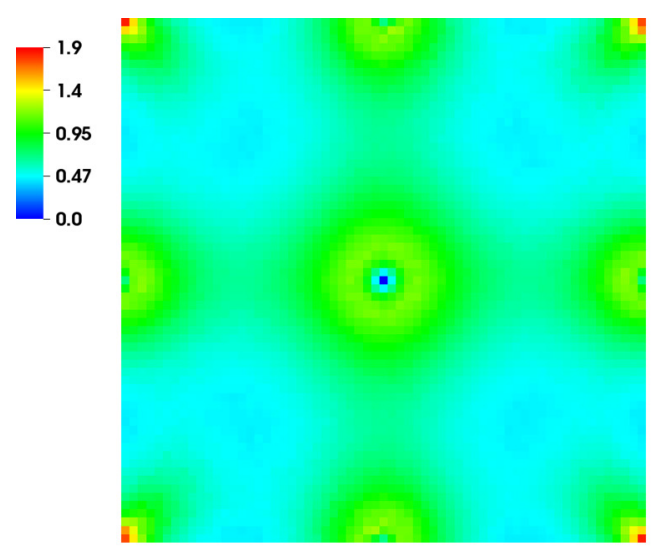

(a) $S_{k}^{\rho \rho}$

\section{APPENDIX B: VERIFICATION OF EQUILIBRIUM SPECTRUM OF FLUCTUATIONS IN TWO-DIMENSIONAL PERIODIC DOMAIN}

Correlations of hydrodynamic variables are calculated using the their fluctuations in Fourier space. For instance, for the density, we have in three dimensions

$$
S_{\mathbf{k}}^{\rho \rho} \equiv S_{\mathbf{k}, 0}^{\rho \rho}=V \lim _{n_{s} \rightarrow \infty}\left\langle\hat{\delta \rho_{\mathbf{k}}^{t_{s}}}\left(\hat{\delta \rho_{\mathbf{k}}^{t_{s}}}\right)^{*}\right\rangle,
$$

where $t_{s}$ is some starting time for the sampling, i.e., $t_{s}=n_{s} \Delta t$, $V$ is the volume of the physical domain, $\delta \rho$ is the fluctuation in density, and the discrete dimensionless wave vector in two dimensions is given by

$$
\mathbf{k}=\left(\frac{2 \pi \kappa_{1}}{N_{1} h}\right) \mathbf{e}_{1}+\left(\frac{2 \pi \kappa_{2}}{N_{2} h}\right) \mathbf{e}_{2}
$$

where $\boldsymbol{\kappa}=\left(\kappa_{1}, \kappa_{2}\right)$ is the wave index. Similarly, we can compute the correlations $S_{\mathbf{k}}^{u_{1} u_{1}}, S_{\mathbf{k}}^{\rho, u_{1}}$, and so on. The magnitudes of autocorrelated quantities such as $S_{k}^{\rho \rho}$ are called structure factors. Theoretical values of the structure factors for single fluids can be derived by linearizing the governing equations [34] and also from statistical mechanics [11]. The expression for the theoretical value of correlations in Fourier space as well as physical space for a binary mixture of ideal gases is provided in the Appendix in [34], which we use here. The numerically calculated values of structure factors are nondimensionalized using their theoretical values; for instance, the normalized density structure factor is given by

$$
S_{\mathbf{k}}^{\rho \rho}=\frac{V}{\left(S_{0}^{\rho \rho}\right)^{d}} \lim _{n_{s} \rightarrow \infty}\left\langle\hat{\delta} \rho_{\mathbf{k}}^{t_{s}}\left(\hat{\delta} \rho_{\mathbf{k}}^{t_{s}}\right)^{*}\right\rangle
$$

where $S_{0}^{\rho \rho}$ is the theoretical value. The fluctuations in hydrodynamic quantities are computed using the guidelines given in [55] to avoid statistical biases. The cross-correlated quantities are normalized by the product of their theoretical values. For instance, the density-velocity correlation is normalized using the theoretical values of both $S_{k}^{\rho \rho}$ and $S_{k}^{u_{1} u_{1}}$ as

$$
S_{k}^{\rho u_{1}}=\frac{V}{\sqrt{\left(S_{0}^{\rho}\right)\left(S_{0}^{u_{1} u_{1}}\right)}} \lim _{n_{s} \rightarrow \infty}\left\langle\hat{\delta} \rho_{k}^{t_{s}}\left(\hat{\delta} v_{k}^{t_{s}}\right)^{*}\right\rangle .
$$

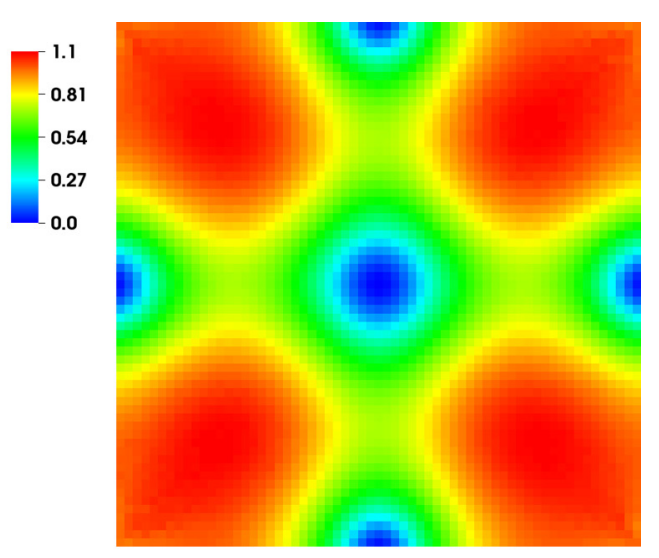

(b) $S_{k}^{E E}$

FIG. 16. Normalized static structure factors. Ideal value is unity. The wave number domain represented here is $\left[-2.45 \times 10^{6}, 2.45 \times 10^{6}\right] \times$ $\left[-2.45 \times 10^{6}, 2.45 \times 10^{6}\right]$ in units of $\mathrm{m}^{-1}$. 


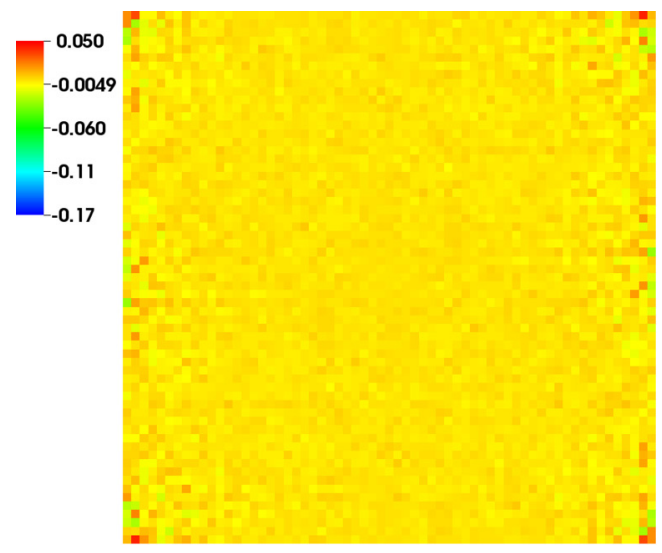

(a) $S_{k}^{\rho u_{1}}$

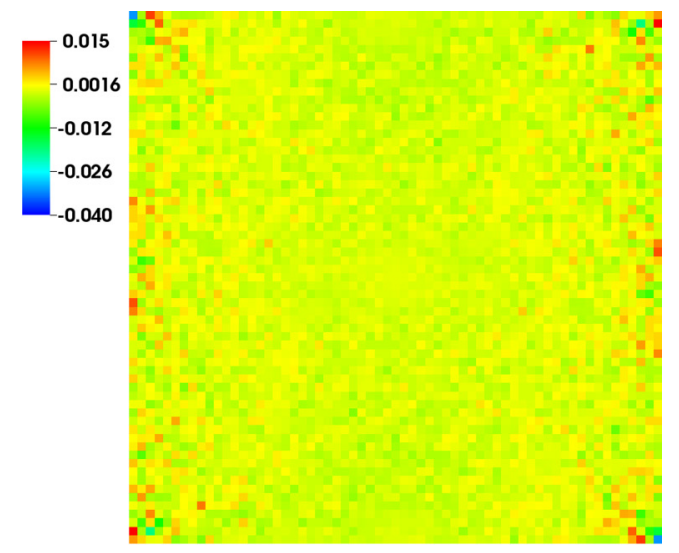

(b) $S_{k}^{u_{1} \rho_{1}}$

FIG. 17. Magnitude of normalized cross-correlations between hydrodynamic quantities. Ideal values are zero. The wave number domain represented here is $\left[-2.45 \times 10^{6}, 2.45 \times 10^{6}\right] \times\left[-2.45 \times 10^{6}, 2.45 \times 10^{6}\right]$ in units of $\mathrm{m}^{-1}$.

Here, we use the static structure factors as the means to verify our choice of $h_{0}$ for the regularization criteria described in Sec. III. Figures 16 and 17 show the plot of the normalized static structure factors and normalized correlations between different hydrodynamic variables, respectively, in a heliumargon mixture at equilibrium with the mass fraction of both set to 0.5 . The cell size was $h_{0}=8 \times 10^{-8} \mathrm{~m}$, and the system temperature was set to STP conditions. The domain was $64 \times$ 64 cells and the time step $\delta t \approx 10^{-12} \mathrm{~s}$. Periodic boundary conditions were used in both the $\mathrm{X}$ and $\mathrm{Y}$ directions, and the system was equilibrated to 40000 steps after which for the statistics we collected over $O\left(10^{6}\right)$ steps.

Figure 16 shows the plots of autocorrelations of the hydrodynamic variables whose ideal value should be unity. We see that for the plot of $S_{k}^{\rho \rho}$, the error compared to the theoretical value is a maximum at the lowest wave numbers because the relaxation time for the largest wavelengths is very high. Here, we note that apart from the four corners of the plot and the blue region in the center of the domain, $S_{k}^{\rho \rho}$ is in a range $0.75-1.25$, which is within an acceptable range of error [34]. We also notice that the results are isotropic owing to the fact that the finite volume method used produces isotropic truncation errors. The density-density correlation has a maximum error because of the lack of a fluctuating term in the mass balance equation owing to which density is driven solely by momentum fluctuation. We see that the errors in $S_{k}^{E E}$ are within $25 \%$ over most of the wave number domain. We observed the same trend for $S_{k}^{\rho_{1} \rho_{1}}, S_{k}^{u_{1} u_{1}}$, and $S_{k}^{\rho E}$, plots of which are not shown for brevity. The normalized cross-correlations except that of $S_{k}^{\rho E}$ should be zero, and Fig. 17 shows that the results obtained are within $8 \%$ error. In conclusion, the verification of the equilibrium fluctuation spectrum leads to our selection of the value of $h_{0}$ that is described in Sec. III to be $8 \times 10^{-8} \mathrm{~m}$.
[1] K. Kadau, C. Rosenblatt, J. L. Barber, T. C. Germann, Z. Huang, P. Carles, B. L. Holian, and B. J. Alder, The importance of fluctuations in fluid mixing, Proc. Natl. Acad. Sci. U.S.A. 104, 7741 (2007).

[2] F. Detcheverry and L. Bocquet, Thermal Fluctuations in Nanofluidic Transport, Phys. Rev. Lett. 109, 024501 (2012).

[3] M. A. Gallis, K. A. Boyles, and G. J. Lebeau, DSMC simulations in support of the Columbia Shuttle orbiter accident investigation, AIP Conf. Proc. 762, 1211 (2005).

[4] A. Vailati, R. Cerbino, S. Mazzoni, C. J. Takacs, D. S. Cannell, and M. Giglio, Fractal fronts of diffusion in microgravity, Nat. Commun. 2, 290 (2011).

[5] R. Delgado-Buscalioni and P. V. Coveney, Hybrid molecularcontinuum fluid dynamics, Philos. Trans. R. Soc. A 362, 1639 (2004).

[6] R. Delgado-Buscalioni and G. De Fabritiis, Embedding molecular dynamics within fluctuating hydrodynamics in multiscale simulations of liquids, Phys. Rev. E 76, 036709 (2007).
[7] S. A. Williams, J. B. Bell, and A. L. Garcia, Algorithm refinement for fluctuating hydrodynamics, Multiscale Model. Simul. 6, 1256 (2008).

[8] P. Español, Stochastic differential equations for non-linear hydrodynamics, Phys. A (Amsterdam, Neth.) 248, 77 (1998).

[9] L. Landau and E. Lifshitz, Hydrodynamic fluctuations, Sov. Phys. JETP 32, 618 (1957).

[10] L. D. Landau and E. M. Lifshitz, in Statistical Physics Part 2, Course on Theoretical Physics Vol. 9 (Pergamon, New York, 1980).

[11] L. Landau and E. Lifshitz, Fluid Mechanics (Pergamon, New York, 1959).

[12] R. F. Fox and G. E. Uhlenbeck, Contributions to non-equilibrium thermodynamics. I. Theory of hydrodynamical fluctuations, Phys. Fluids 13, 1893 (1970).

[13] G. Kelly and M. Lewis, Hydrodynamic fluctuations, Phys. Fluids 14, 1925 (1971).

[14] C. Cohen, J. W. H. Sutherland, and J. M. Deutch, Hydrodynamic correlation functions for binary mixtures, Phys. Chem. Liq. 2, 213 (1971). 
[15] J. Nieuwoudt and B. Law, Theory of light scattering by a nonequilibrium binary mixture, Phys. Rev. A 42, 2003 (1990).

[16] J. O. de Zarate and J. Sengers, Hydrodynamic Fluctuations in Fluids and Fluid Mixtures (Elsevier, Amsterdam, 2006).

[17] A. García and C. Penland, Fluctuating hydrodynamics and principal oscillation pattern analysis, J. Stat. Phys. 64, 1121 (1991).

[18] M. Mareschal, M. M. Mansour, G. Sonnino, and E. Kestemont, Dynamic structure factor in a nonequilibrium fluid: A moleculardynamics approach, Phys. Rev. A 45, 7180 (1992).

[19] M. M. Mansour, A. L. Garcia, G. C. Lie, and E. Clementi, Fluctuating Hydrodynamics in a Dilute Gas, Phys. Rev. Lett. 58, 874 (1987).

[20] P. Espanol, The micro-meso connection also known as nonequilibrium statistical mechanics also known as the theory of coarse-graining, Courant Institute of Mathematical Sciences, report, 2013 (unpublished).

[21] H. C. Öttinger and M. Grmela, Dynamics and thermodynamics of complex fluids. II. Illustrations of a general formalism, Phys. Rev. E 56, 6633 (1997).

[22] M. Brouilette, The Richtmyer-Meshkov instability, Annu. Rev. Fluid Mech. 34, 445 (2002).

[23] S. I. Abarzhi, Review of theoretical modeling approaches of Rayleigh-Taylor instabilities and turbulent mixing, Philos. Trans. R. Soc. A 368, 1809 (2010).

[24] D. H. Sharp, An overview of Rayleigh-Taylor instability, Phys. D (Amsterdam, Neth.) 12, 3 (1984).

[25] W. Alda, W. Dzwinel, J. Kitowski, J. Moscinski, M. Pogoda, and D. A. Yuen, Complex fluid-dynamical phenomena modeled by large-scale molecular-dynamics simulations, Comput. Phys. 12, 595 (1998).

[26] G. Dimonte, D. L. Youngs, A. Dimits, S. Weber, M. Marinak, S. Wunsch, C. Garasi, A. Robinson, M. J. Andrews, P. Ramaprabhu, A. C. Calder, B. Fryxell, J. Biello, L. Dursi, P. MacNeice, K. Olson, P. Ricker, R. Rosner, F. Timmes, H. Tufo, Y. N. Young, and M. Zingale (Alpha-Group Collaboration), A comparative study of the turbulent Rayleigh-Taylor instability using high-resolution three-dimensional numerical simulations, Phys. Fluids 16, 1668 (2004).

[27] S. B. Dalziel, P. F. Linden, and D. L. Youngs, Self similarity and internal structure of turbulence induced by Rayleigh Taylor instability, J. Fluid Mech. 399, 1 (1999).

[28] J. L. Barber, K. Kadau, T. C. Germann, and B. J. Alder, Initial growth of the Rayleigh-Taylor instability via molecular dynamics, Eur. Phys. J. B 64, 271 (2008).

[29] A. L. Garcia, M. M. Mansour, G. C. Lie, and E. Cementi, Numerical integration of the fluctuating hydrodynamic equations, J. Stat. Phys. 47, 209 (1987).

[30] J. B. Bell, A. L. Garcia, and S. A. Williams, Numerical methods for the stochastic Landau-Lifshitz Navier-Stokes equations, Phys. Rev. E 76, 016708 (2007).

[31] A. Donev, E. Vanden-Eijnden, A. Garcia, and J. Bell, On the accuracy of finite-volume schemes for fluctuating hydrodynamics, Commun. Appl. Math. Comput. Sci. 5, 149 (2010).

[32] F. Balboa, J. B. Bell, R. Delgado-Buscalioni, A. Donev, T. G. Fai, B. E. Griffith, and C. S. Peskin, Staggered schemes for fluctuating hydrodynamics, Multiscale Model. Simul. 10, 1369 (2012).
[33] J. Bell, A. Garcia, and S. Williams, Computational fluctuating fluid dynamics, ESAIM Math. Modell. Numer. Anal. 44, 1085 (2010).

[34] K. Balakrishnan, A. L. Garcia, A. Donev, and J. B. Bell, Fluctuating hydrodynamics of multi-species nonreactive mixtures, Phys. Rev. E 89, 013017 (2014).

[35] A. K. Bhattacharjee, K. Balakrishnan, A. L. Garcia, J. B. Bell, and A. Donev, Fluctuating hydrodynamics of multispecies reactive mixtures, J. Chem. Phys. 142, 224107 (2015).

[36] M. A. Gallis, T. P. Koehler, J. R. Torczynski, and S. J. Plimpton, Direct simulation Monte Carlo investigation of the RichtmyerMeshkov instability, Phys. Fluids 27, 084105 (2015).

[37] V. V. Zhakhovskii, S. V. Zybin, S. I. Abarzhi, and K. Nishihara, Atomistic dynamics of the Richtmyer-Meshkov instability in cylindrical and planar geometries, AIP Conf. Proc. 845, 433 (2006).

[38] B. Collins and J. Jacobs, PLIF flow visualization and measurements of the Richtmyer-Meshkov instability of an air/SF6 interface, J. Fluid Mech. 464, 113 (2002).

[39] J. Hirshfelder, C. Curtis, and R. Bird, Molecular Theory of Gases and Liquids (John Wiley and Sons, New York, 1954).

[40] R. Chandrasekhar, Stochastic problems in physics and astronomy, Mod. Phys. 15, 1 (1943).

[41] A. Donev, T. G. Fai, and E. Vanden-Eijnden, A reversible mesoscopic model of diffusion in liquids: From giant fluctuations to Fick's law, J. Stat. Mech.: Theor. Exp. (2014) P04004.

[42] N. J. Zabusky, Vortex paradigm for accelerated inhomgeneous flows: visiometrics for the Rayleigh-Taylor and RichtmyerMeshkov environments, Annu. Rev. Fluid Mech. 31, 495 (1999).

[43] R. D. Richtmyer, Taylor instability in shock acceleration of compressible fluids, Commun. Pure Appl. Math. 13, 297 (1960).

[44] E. E. Meshkov, Instability of the interface of two gases accelerated by a shock wave, Fluid Dyn. 4, 101 (1972).

[45] P. A. Thompson and G. S. Beavers, Compressible-Fluid Dynamics (McGraw-Hill, New York, 1972), p. 366.

[46] O. Sadot, L. Erez, U. Alon, D. Oron, L. A. Levin, G. Erez, G. BenDor, and D. Shvarts, Study of Nonlinear Evolution of SingleMode and Two-Bubble Interaction under Richtmyer-Meshkov Instability, Phys. Rev. Lett. 80, 1654 (1998).

[47] C. E. Niederhaus and J. W. Jacobs, Experimental study of the Richtmyer-Meshkov instability of incompressible fluids, J. Fluid Mech. 485, 243 (2003).

[48] V. N. Goncharov, Analytical Model of Nonlinear, Single-Mode, Classical Rayleigh-Taylor Instability at Arbitrary Atwood Numbers, Phys. Rev. Lett. 88, 134502 (2002).

[49] S.-I. Sohn, Simple potential-flow model of Rayleigh-Taylor and Richtmyer-Meshkov instabilities for all density ratios, Phys. Rev. E 67, 026301 (2003).

[50] R. P. Drake, High-energy-density physics, Phys. Today 63(6), 28 (2010).

[51] R. Samtaney, A method to simulate linear stability of impulsively accelerated density interfaces in ideal-MHD and gas dynamics, J. Comput. Phys. 228, 6773 (2009).

[52] F. Cobos-Campos and J. G. Wouchuk, Analytical scalings of the linear Richtmyer-Meshkov instability when a rarefaction is reflected, Phys. Rev. E 96, 013102 (2017). 
[53] B. Duenweg and A. J. C. Ladd, Lattice Boltzmann simulations of soft matter systems, in Advanced Computer Simulation Approaches for Soft Matter Sciences III, Advances in Polymer Science Vol. 221, edited by C. Holm and K. Kremer (Springer, Berlin, 2008).
[54] S. Delong, B. E. Griffith, E. Vanden-Eijnden, and A. Donev, Temporal integrators for fluctuating hydrodynamics, Phys. Rev. E 87, 033302 (2013).

[55] A. Garcia, Estimating hydrodynamic quantities in the presence of microscopic fluctuations, Commun. Appl. Math. Comput. Sci. 1, 53 (2006). 